\title{
Auxiliary Constructs for Proving Liveness in Compassion Discrete Systems
}

\author{
Teng Long and Wenhui Zhang \\ State Key Laboratory of Computer Science \\ Institute of Software, Chinese Academy of Sciences, Beijing, China \\ \{longteng,zwh\}@ios.ac.cn
}

\begin{abstract}
For proving response properties for systems with compassion requirements, a deduction rule is introduced in [13]. In order to use the rule, auxiliary constructs are needed. They include helpful assertions and ranking functions defined on a well-founded domain. Along the line of the work [2] that computes ranking functions for response properties for systems with justice requirements, we develop an approach which extends that of [2] for computing ranking functions for systems with compassion requirements. We illustrate the use of the approach on three examples.
\end{abstract}

\section{Introduction}

Model checking is a main verification technique for finite state systems, and has been successfully applied to proving the correctness of hardware and software designs. However, in the general case, the real systems in the world may be (practically) infinite, such that model checking cannot be directly used to prove the correctness of such systems.

The concept of abstraction helps enhancing the applicability of model checking to infinite systems. Predicate abstraction $[8,4,5]$, has been useful for verification of safety properties of infinite systems, and for verification of liveness properties. Ranking abstraction has been introduced in $[9,3,10]$ recognizing that the usual state abstraction is often inadequate in order to capture liveness properties, in which compassion requirements are introduced into the abstract system such that the abstraction preserves the liveness properties under consideration.

One of the common features of these two methods is that we need to extract auxiliary constructs in order to make the methods successful in proving safety and liveness properties. In the former case, one needs to construct invariants and in the latter, one needs to construct ranking functions.

Our focus is on methods for computing ranking functions for proving liveness properties. Invisible ranking introduced in $[6]$ is one such method for automatically generating helpful assertions and ranking functions for proving liveness properties in systems with justice requirements. The method was then extended to handle a large class of problems by relaxing restrictions requiring that the helpful assertions and ranking functions only depend on the local states of a process [7]. For dealing with ranking abstraction in proving liveness properties 
in systems with justice requirements, an approach is presented in [2] based on graph manipulation for generating helpful assertions and ranking functions.

Along this line of research, our approach presented in this paper extends that of [2] in order to be able to compute ranking functions for proving liveness properties in sequential and concurrent programs with compassion requirements including those introduced with ranking abstraction and those stated explicitly in the compassion discrete system. Our approach may as well be used for proving liveness properties with the use of predicate abstraction (when ranking abstraction does not provide additional useful compassion requirements).

The rest of this paper is organized as follows. In Section 2 we introduce the basic concepts used in the approach. It includes the computational model FDS (fair discrete system) and CDS (compassion discrete system) with its related notions of fairness, the rule RESPONSE [13] for the deductive proof of response properties of CDS. Section 3 presents the approach for computing the auxiliary constructs, and Section 4 illustrates the application of the approach on three examples. Finally, concluding remarks are contained in Section 5.

\section{Preliminaries}

We introduce the computational model with fairness requirements [11], and the rule for proving response properties [13].

\subsection{Computational Model}

A fair discrete system (FDS) is a quintuple $D=\langle V, \Theta, \rho, J, C\rangle$ where the components are as follows.

- $V$ : A finite set of typed system variables, containing data and control variables. A set of states (interpretation) over $\mathrm{V}$ is denoted by $\Sigma$. For a state s and a system variable $v \in V$, we denote by $\mathrm{s}[\mathrm{v}]$ the value assigned to $\mathrm{v}$ by the state s.

- $\Theta$ : The initial condition - an assertion (state formula) characterizing the initial states.

- $\rho$ : The transition relation - an assertion $\rho\left(V, V^{\prime}\right)$, relating the variables in state $s \in \Sigma$ to the $V^{\prime}$ in a D-successor state $s^{\prime} \in \Sigma$.

$-J$ : A set of justice requirements (weak fairness). The justice requirement $J \in \mathcal{J}$ is an assertion which guarantee that every computation should include infinitely many states satisfying $J$.

$-C:$ A set of compassion requirements (strong fairness). The compassion requirement $\langle p, q\rangle \in C$ is a pair of assertions, which guarantee that every computation should include either only finitely many p-states, or infinitely many q-states.

Computation A computation of $D$ is an infinite sequence of state $\sigma: s_{0}, s_{1}, s_{2}, \ldots$, satisfying the following requirements: (1) $s_{0} \models \Theta$. (2) For each $\mathrm{j}=0,1, \ldots$, the state $s_{j+1}$ is in a D-successor of the state $s_{j}$. For each $v \in V$, we interpret $v$ as $s_{l}[v]$ and $v^{\prime}$ as $s_{l+1}[v]$, that is $\left\langle s_{l}, s_{l+1}\right\rangle \models \rho\left(V, V^{\prime}\right)$. 
Justice A computation $\sigma$ is just, if $\sigma$ contains infinitely many occurrences of $J$-states for every $J \in \mathcal{J}$. A justice discrete system (JDS) is an FDS with no compassion requirements.

Compassion A computation $\sigma$ is compassionate, if $\sigma$ contains only finitely many p-states, or $\sigma$ contains infinitely many q-states, for every $\langle p, q\rangle \in C$. A compassion discrete system (CDS) is an FDS with no justice requirements.

\subsection{Proof Rule for Response Properties}

The problem is that given an FDS $D$ and a response property of the form $p \Rightarrow \diamond q$, where $p$ and $q$ are assertions, we want to prove the following statement

$$
D \models(p \Rightarrow \diamond q)
$$

Since an FDS is equivalent to a $\mathrm{CDS}^{1}$, it is sufficient to consider CDS only. For proving response properties over a CDS, the rule RESPONSE [13] was developed for systems with compassion requirements (this rule is hereafter referred to as C-RESPONSE for emphasizing that it involves compassion requirements). The rule is shown in Fig. 1.

Let $p, q$ be assertions.

Let $\mathcal{A}:(W, \succ)$ be a well-founded domain.

Let $\left\{\varphi_{i} \mid i \in\{1, \ldots, n\}\right\}$ be a set of assertions.

Let $\left\{F_{i}=\left\langle p_{i}, q_{i}\right\rangle \mid i \in\{1, \ldots, n\}\right\}$ be a set of compassion requirements.

Let $\left\{\Delta_{i}: \Sigma \rightarrow W \mid i \in\{1, \ldots, n\}\right\}$ be a set of ranking functions.

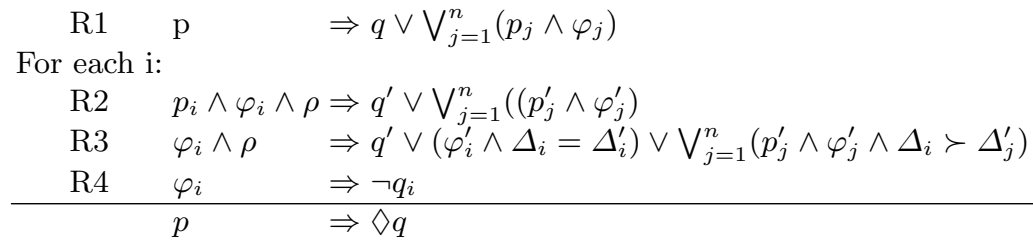

Fig. 1. Proof Rule: C-RESPONSE

The use of the rule requires: a well-founded domain $\mathcal{A}$, and for each compassion requirement $\left\langle p_{i}, q_{i}\right\rangle$, a helpful assertion $\varphi_{i}$ and a ranking function $\Delta_{i}: \Sigma \mapsto$ $W$ mapping states of $D$ to elements of $\mathcal{A}$.

$\mathrm{R} 1$ requires that any $p$-state is either a goal state (i.e., a $q$-state), or a $\left(p_{i} \wedge \varphi_{i}\right)$ state for some $i \in\{1, \ldots, n\}$. R2 requires that any step from a $\left(p_{i} \wedge \varphi_{i}\right)$-state moves either directly to a $q$-state, or to another $\left(p_{j} \wedge \varphi_{j}\right)$-state, or stays at a state of the same type (i.e., a $\left(p_{i} \wedge \varphi_{i}\right)$-state). R3 requires that any step from

\footnotetext{
${ }^{1}$ The justice requirement can be expressed as the degenerate compassion requirement $\langle 1, J\rangle$, where 1 denotes the assertion True which holds at every state.
} 
a $\varphi_{i}$-state moves either directly to a $q$-state, or to another $\left(p_{j} \wedge \varphi_{j}\right)$-state with decreasing rank, or stays at a state of the same type with the same rank. R4 together with the previous rules guarantees that if an execution does not satisfy $\diamond q$, then it violates the compassion requirement.

\section{Proving a Response Property}

Given a $\operatorname{CDS} D$ and a response property $\psi: p \Rightarrow \diamond q$. In order to be able to use the proof rule C-RESPONSE for proving the property, we have to define a well-founded domain $\mathcal{A}$, and for each compassion requirement $\left\langle p_{i}, q_{i}\right\rangle$, define a helpful assertion $\varphi_{i}$ and a ranking function $\Delta_{i}: \Sigma \mapsto W$ mapping states of $D$ to elements of $\mathcal{A}$.

The phases for proving $D \models \psi$ including those of computing the helpful assertions and ranking functions are as follows:

(1) use ranking abstraction $[9,3,2]$ and construct $D^{\alpha}$ and $\psi^{\alpha}$ from $D$ and $\psi$, and then construct a pending graph [2] based on $D^{\alpha}$.

(2) construct an initial rank for each node (except the goal state) of the pending graph and a set of helpful compassion requirements associated to each of these nodes of the pending graph.

(3) construct an abstract graph from the pending graph, such that each node in the abstract graph represents a subset of the nodes of the pending graph, then construct $\mathcal{A}$, and for each node, construct $\varphi_{i}$ and $\Delta_{i}$, and make an association of some $F_{i}=\left(p_{i}, q_{i}\right) \in \mathcal{F}$ to the node. Note that according to the construction, one $F_{i}$ may correspond to several abstract nodes.

(4) use the helpful constructs to prove the response property $D \models \psi$ by the proof rule.

\subsection{Ranking Abstraction and Pending Graph}

This step is carried out according to the technique of ranking abstraction $[9,3$, 2] and pending graph [2].

Ranking abstraction, as explained in [2], is a method of augmenting the concrete program by a non-constraining progress monitor, which measures the progress of program execution, relative to a given ranking function. In order to distinguish this kind of ranking functions from the ranking functions in the proof rule C-RESPONSE, we call this kine of ranking functions ARFs (augmenting ranking functions) in the sequel. Once a program is augmented, a conventional state abstraction can be used. In such a way, the state abstraction can preserve the ability to monitor progress in the abstract system.

For a system $S=\langle V, \Theta, \rho, J, C\rangle$ (in which $J$ is empty when CDS is considered) and a well-founded domain $(W, \prec)$, let $\delta$ be an ARF over $W$, let $d e c_{\delta}$ be a fresh variable, the augmentation of $S$ by $\delta$ is

$$
S+\delta:\left\langle V \cup\left\{d e c_{\delta}\right\}, \Theta, \rho \wedge \rho_{\delta}, J, C \cup\left\{\left(\operatorname{dec}_{\delta}>0, \operatorname{dec}_{\delta}<0\right)\right\}\right\rangle
$$


where $\rho_{\delta}$ is defined by

$$
\operatorname{dec}_{\delta}^{\prime}= \begin{cases}1 & \delta \succ \delta^{\prime} \\ 0 & \delta=\delta^{\prime} \\ -1 & \text { otherwise }\end{cases}
$$

A system may be augmented with a set of ARFs $\left\{\delta_{1}, \ldots, \delta_{k}\right\}$. Then predicate abstraction may be applied. In the predicate abstraction, it is not necessary to abstract variables of the form $d e c_{\delta}$ since it ranges over the finite domain $\{-1,0,1\}$, and the abstraction preserves the compassion requirement $\left(\operatorname{dec}_{\delta}>\right.$ $\left.0, \operatorname{dec}_{\delta}<0\right)$.

Assuming that we already have an abstract program $D^{\alpha}$ from $D$ constructed by the above process with the abstraction map $\alpha$, a pending graph [2] is then constructed from $D^{\alpha}$. Let us denote the graph by $G=\langle N, E\rangle$. The set of nodes $N$ are those satisfying pend $\vee g$ where pend characterizes the states reachable from a $p$-state by a $q$-free path, and $g$ is a $q^{\alpha}$-state reachable from a pending state in one step. The set of edges $E$ consists of all transitions connecting two pending states and the edges connecting pend nodes to the goal node $g$.

The set of nodes of $G$ may be written as $\left\{S_{0}, S_{1}, \ldots, S_{m}\right\}$ where $S_{0}=g$ is the goal state and $S_{1}, \ldots, S_{m}$ are pending states. This is the starting point of our algorithm for computing the helpful constructs for the proof rule.

\subsection{Helpful Compassion Requirements and Initial Ranks}

The ranking functions in the abstract program are represented as a mapping $N \rightarrow T U P L E S$, where TUPLES is the type of lexicographic tuples whose elements are either natural numbers or ARFs. For simplicity, we call such a tuple as a "rank". Let $R_{l}$ and $H_{l}$ be respectively the rank and the list of helpful compassion requirements for $S_{l} \in N$. For convenience, we write $q$ for $q^{\alpha}$, and similarly for other formulas and constructions. Let $F_{1}, \ldots, F_{n}$ be the original compassion requirements, and $F_{n+1}, \ldots, F_{n^{\prime}}$ be the dec-requirements (the compassion requirements introduced by the ranking abstraction). The procedure for computing $R_{l}$ and $H_{l}$ (which are initially empty) is described in Algorithm 1. The main functions are explained as follows.

decompose $(G)$ The graph $G$ is decomposed into a set of MSCCs (maximal strongly connected components), denoted $C_{0}, \ldots, C_{k}$. They are ordered such that if $C_{i}$ is reachable from $C_{j}$, then $i<j$. For MSCCs not connected to each other, their indices may be in an arbitrary order.

violate $\left(C_{i}, F_{j}\right)$ The MSCC (may be the trivial one) $C_{i}$ violates the compassion requirement $F_{j}=\left(p_{j}, q_{j}\right)$, if $p_{j}$ is satisfied by some node of the MSCC and $q_{j}$ is not satisfied by any node of the MSCC.

$\operatorname{term}\left(F_{j}\right)$ For a dec-requirement $F_{j}$ of the form $\left\langle\operatorname{dec}_{\delta}>0, \operatorname{dec} \delta<0\right\rangle, \operatorname{term}\left(F_{j}\right)=$ $\delta$. 


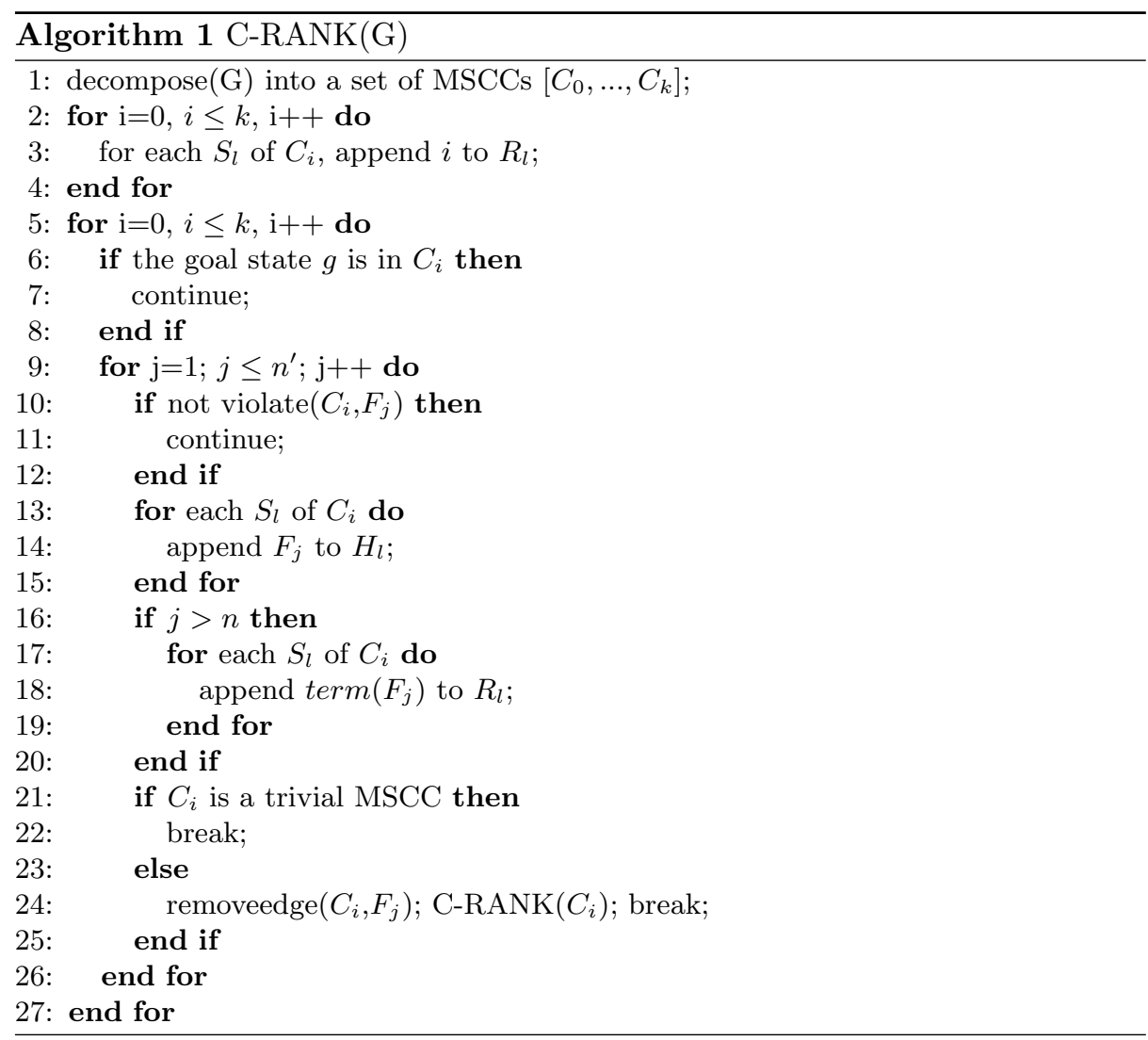

removeedge $\left(C_{i}, F_{j}\right)$ Given an MSCC $C_{i}$ and a compassion requirement $F_{j}=$ $\left(p_{j}, q_{j}\right)$, this procedure modifies the MSCC in such a way that one node satisfying $p_{j}$ is identified and all incoming edges of such a node in this MSCC are removed.

\subsection{Abstract Nodes, Helpful Assertions and Ranks}

According to $H_{l}$, we construct the abstract nodes as an assertion $\Phi$ by grouping together certain nodes that need satisfying the same helpful compassion requirements. Let $G^{\prime}$ be the abstract graph, initially empty, i.e., $G^{\prime}=(\{\},\{\})$. The procedure for constructing $G^{\prime}$ is described in Algorithm 2. The main functions are explained as follows.

$\operatorname{subgraph}\left(G, F_{i}\right)$ In the assignment $W_{i}=\operatorname{subgraph}\left(G, F_{i}\right)$, the variable $W_{i}$ is a local variable used to hold a subset of nodes (a subgraph). The nodes of the subgraph is constructed according to $F_{i}$ (non-dec-requirements) as follows: $S_{l} \in W_{i}$ iff $F_{i} \in H_{l}$. Then $W_{i}$ is considered as a derived subgraph of $G$ with the nodes as specified. 


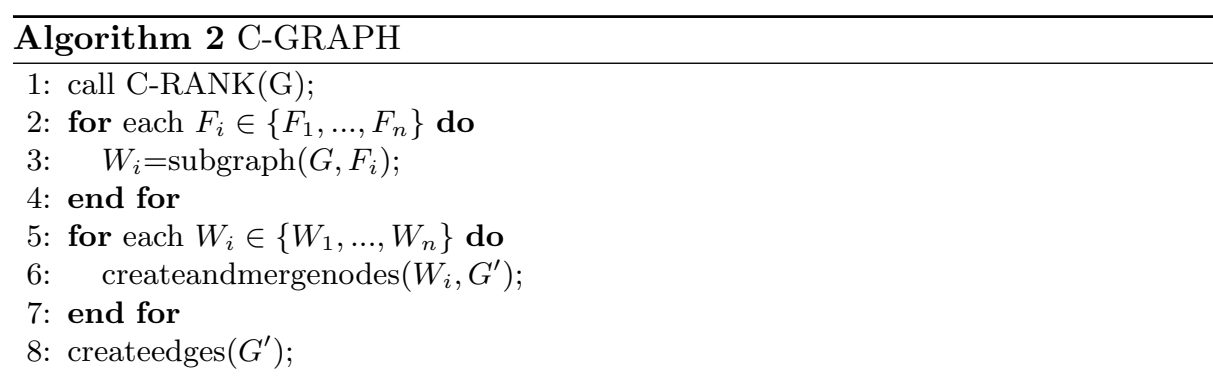

createandmergenodes $\left(W_{i}, G^{\prime}\right)$ (1) $W_{i}$ may contain several different MSCCs violating $F_{i}$. For each such MSCC in $W_{i}$, a node representing this MSCC is created and added to $G^{\prime}$. The rank $\Delta_{l}$ of the abstract node $\Phi_{l}$ is assigned the rank obtained before the MSCC is split into smaller MSCCs. (2) For the nodes created previously, they are merges according to the following condition: the states that the nodes represent differ only in the dec-variables introduced in the ranking abstraction. Then the rank of the abstract node is assigned the lowest rank of the nodes represented by the abstract node. (3) For each node $\Phi_{l}$ in the final abstract graph $G^{\prime}$, the concrete helpful assertion $\varphi_{l}=\alpha^{-1}\left(\Phi_{l}\right)$ is obtained by concretizing the abstract nodes (viewed as abstract assertions, by making correspondence between formulas and sets of states).

createedges $\left(G^{\prime}\right)$ For each pair of nodes $\Phi$ and $\Phi^{\prime}$ such that $\Phi^{\prime} \not \subset \Phi$, if some node of $\Phi$ is connected to some of $\Phi^{\prime}$, an edge from $\Phi$ to $\Phi^{\prime}$ is created.

\subsection{Claim of the Helpful Constructions}

For each compassion requirement $\left(p_{i}, q_{i}\right)$, several abstract states may be associated. We consider the set of compassion requirements as a multiset, such that one compassion requirement $\left(p_{i}, q_{i}\right)$ has the number of occurrences matching the number of associated abstract states. Then each (occurrence of a) compassion requirement corresponds to one abstract state (with a rank and a helpful assertion) associated with it. These helpful constructs are then to be used in the proof rule for proving the response property $D \models \Psi$.

Ranking Core For the correctness, we assume that every ranking function in the ranking abstraction is chosen to be a variable. Such a set of variables (representing a set of ranking functions) are called ranking core $\mathcal{R}$ [9]. It is easily seen that the proof of the correctness of the above algorithms with this assumption can be extended to ranking functions that are arithmetic terms. The abstraction of $D$ according to the abstraction map $\alpha$ and the ranking core $\mathcal{R}$ is denoted $\mathcal{D}^{\mathcal{R}, \alpha}$.

Main Claim Let CDS D , a ranking core $\mathcal{R}$, an abstraction mapping $\alpha$ and the property $\Psi$ be given. Let the assertions $\varphi_{i}$ and ranking functions $\Delta_{i}$ be those extracted by $C$-GRAPH. If $\mathcal{D}^{\mathcal{R}, \alpha}=\Psi^{\alpha}$ then $R_{1}-R_{4}$ of the rule $C$-RESPONSE 
are provable with the extracted helpful constructs (with the set of compassion requirements reorganized as a suitable multiset).

Correctness The correctness is then established by analyzing the different steps in the construction of the helpful constructs.

Let $\Delta>\Delta^{\prime}$ (similarly $R>R^{\prime}$ ), where $\Delta, \Delta^{\prime}$ represent ranks and $R, R^{\prime}$ represent ranks, denote that $\Delta$ appear after $\Delta^{\prime}$ according to the lexicographic order. Let $\Delta \succ_{E} \Delta^{\prime}$ denote that $\Delta$ appear after $\Delta^{\prime}$ according to the lexicographic order with the following conditions: (1) each variable $x$ of the ranking core $\mathcal{R}$ appearing in $\Delta^{\prime}$ are replaced by $x^{\prime},(2)$ the lexicographic order is augmented by an environment $E$ that specifies whether $x>x^{\prime}$ or $x=x^{\prime}$. The environment $E$ may be replaced by a state $S$ that reflects whether the value of a variable is decreased when the program moves to the state $S$.

Claim 1 Let $S_{i}$ and $S_{j}$ be states in the pending graph. The following properties hold.

$P_{1}$. If $\Delta_{i} \succ_{S_{j}} \Delta_{j}$ and $\Delta_{j}>\Delta_{k}$, then $\Delta_{i} \succ_{S_{j}} \Delta_{k}$.

$P_{2}$. If the states $S_{i}$ and $S_{j}$ agree on the values of their non-dec variables, then they have the same set of successors.

$P_{1}$ is true according to the definition. $P_{2}$ is true according to the construction of the pending graph. These properties are the same as those stated in [2].

Claim 2 Let $R_{i}$ and $R_{j}$ be the associated ranks of $S_{i}$ and $S_{j}$. Then on successful termination of $C$-RANK, the following properties hold.

$P_{3}$. For every two states $S_{i}, S_{j}$ belonging to different MSCCs such that $S_{i}$ is connected to $S_{j}$, there is a rank decrease $R_{i} \succ_{S_{j}} R_{j}$.

$P_{4}$. For every two states $S_{i}, S_{j}$ belonging to one MSCC such that $S_{i}$ is connected to $S_{j}$, there is no rank decrease only if the MSCC violates some compassion requirement $\left(p_{k}, q_{k}\right)$ (non-dec-requirements) and there is at least a state $S_{k}$ which $S_{k}=p_{k}$, or the MSCC does not violate any compassion requirement.

$P_{3}$ follows from the decomposition of the pending graph into MSCCs. $P_{4}$ follows from the way MSCCs being modified when they do not satisfy some compassion requirements.

Claim 3 Let $\Delta_{i}$ and $\Delta_{j}$ be the associated ranks of $\Phi_{i}$ and $\Phi_{j}$. Then on termination of C-GRAPH, the following properties hold.

$P_{5}$. If $\Phi_{i}$ is connected to $\Phi_{j}$ in the abstract graph and $S \in \Phi_{j}$, then there is a $\Phi_{k}$ such that $S \in \Phi_{k}, \Delta_{i} \succ_{S} \Delta_{k}$ and $S \models p_{k}$ where $\left(p_{k}, q_{k}\right)$ is the compassion requirement violated by the MSCC represented by $\Phi_{k}$.

$P_{6}$. Let $s[\Delta]$ be $\Delta$ with the variables replaced by their value in the state $s$.

If concrete states $s, s^{\prime}$ satisfy $s \models \varphi_{i}$, and $s^{\prime} \models \varphi_{j}, i \neq j$ and $s^{\prime}$ is a $D$ successor of $s$, then there is a $\varphi_{k}$ such that $s^{\prime} \models \varphi_{k} \wedge p_{k}$ and $s\left[\Delta_{i}\right] \succ s^{\prime}\left[\Delta_{k}\right]$. 
$P_{5}$ follows from the construction of the abstract graph. $\Phi_{k}$ in $P_{5}$ is necessarily a superset of $\Phi_{j}$ (i.e., $\Phi_{j} \subseteq \Phi_{k}$ ), when $\Phi_{k}$ is considered as a set of nodes of the pending graph. $P_{6}$ follows from $P_{5}$ by the soundness of the abstraction.

Proof of the main claim. Let $\Phi_{0}=g, \Phi_{1}, \ldots, \Phi_{n}$ be the nodes in the abstract graph, and $\varphi_{i}, \Delta_{i},\left(p_{i}, q_{i}\right)$ be the helpful assertion, rank, and compassion requirement (which has been reorganized into a multiset that matches the number of $\Phi_{i}$ ) associated to $\Phi_{i}$ for $i=1, \ldots, n$. Assume $D^{\alpha}=\Psi^{\alpha}$. (1) Since $\Psi^{\alpha}$ is true, the disjunction of the abstract states $g, \Phi_{1}, \ldots, \Phi_{n}$ covers the states in the pending graph. By the correctness of the abstraction, $g \vee \bigvee_{i=1}^{l} \varphi_{i}$ covers the state space represented by the pending graph. The a $p$-state is either the goal state $g$ or a state with a progress requirement, i.e. $p_{i} \wedge \varphi_{i}$ for some $i \in\{1, \ldots, n\}$. (2) Similarly, successor states of such a state (excluding $g$ ) also satisfy the same condition. (3) The correctness of $R_{3}$ follows from property $P_{6}$. (4) $R_{4}$ is guaranteed by the construction of $\varphi_{i}$, since each $\varphi_{i}$ represents an MSCC (or a collection of MSCCs when they are merged) violating the compassion requirement $\left(p_{i}, q_{i}\right)$.

\subsection{Discussion}

Previous works in this direction of research include using deduction rules with weak fairness (justice) requirements to prove liveness properties of sequential or simple concurrent programs. They depend on Dec-requirements to decide the ranks of states in just MSCC. Our approach concerns deductive rule with strong fairness requirements to prove liveness properties of more complex concurrent programs. It depends on compassion requirements to decide the ranks of states in MSCC. A comparison of the algorithm RANK-JUST-GRAPH [2] and C-GRAPH is given in Fig.2.

\begin{tabular}{|l|l|l|}
\hline Algorithm & RANK-JUST-GRAPH & C-GRAPH \\
\hline Deductive Rule & JUST-RESPONSE & C-RESPONSE \\
\hline Fairness of Systems & Weak fairness & Fairness \\
\hline Control of Unfair Loops & $\begin{array}{l}\text { Depend on Dec- } \\
\text { requirements to decide the } \\
\text { ranks of states in MSCC }\end{array}$ & $\begin{array}{l}\text { Depend on compassion } \\
\text { requirements to decide the } \\
\text { ranks of states in MSCC }\end{array}$ \\
\hline
\end{tabular}

Fig. 2. Algorithms for Computing Auxiliary Constructs

\section{Examples}

We illustrate the application of the approach on three programs:

- MUX-SEM, a concurrent program for mutual exclusion [1]. This example is supposed to show the approach applied on a verification problem with predicate abstraction. 
- COND-TERM, a sequential program with a non-deterministic choice of the values of a variable [13].

This example is supposed to show the approach applied on a verification problem with predicate abstraction in which some Dec-requirement is introduced in the abstraction phase. The pending graph includes two kinds of uncompassionate loops, one violating the given compassion and the other violating the compassion introduced in the abstraction phase.

- UP-DOWN, a concurrent program with only justice requirements [12].

This example is supposed to show some differences and similarities between this approach and the approach presented in [2] when compassion requirements in the system are restricted to justice requirements.

\subsection{Example 1: MUX-SEM}

Fig. 3 shows the program MUX-SEM. Let $a t l_{i}[j]$ denotes that process $j$ is at $l_{i}$ (of process $j$ ). The response property we wish to establish is at $l_{2}[1] \Rightarrow \diamond a t l_{3}[1]$. The just requirements are $\neg a t_{-} l_{4}[j]$ and $\neg a t_{l_{3}}[j]$. The compassion requirement is $F_{1}=\left\langle a t_{-} l_{2}[1] \wedge y=1, a t_{-} l_{3}[1]\right\rangle$. The just requirements are special compassion requirements formulated as $\left\langle 1, \neg a t_{-} l_{4}[i]\right\rangle$ for $i \in\{1, \ldots, n\}$ and $\left\langle 1, \neg a t_{-} l_{3}[i]\right\rangle$ for $i \in\{1, \ldots, n\}$.

$$
\begin{gathered}
\text { local } y: \text { boolean init } y=1 ; \\
\|_{i=1}^{n} P[i]::\left[\begin{array}{c}
\text { loop } \text { forever do } \\
l_{1}: \text { Noncritical } \\
l_{2}: \text { request } y \\
l_{3}: \text { Critical } \\
l_{4}: \text { release } y
\end{array}\right]
\end{gathered}
$$

Fig. 3. Program MUX-SEM

The abstraction mapping $\alpha$ is defined by:

$$
\alpha: \Pi=\pi, \Pi_{3}=\pi_{3}, \Pi_{4}=\pi_{4}, Y=(y>0)
$$

where $\Pi$ is a function with range $\{1,2,3,4\}$ (and the domain being the system states). $\Pi=i$ denotes that at $l_{i}[1]$ is true, for $i \in\{1, \ldots, 4\}$. $\Pi_{k}$ is a function with range $\{0,1\}$ and it is 1 iff the following is true:

$$
\bigvee_{j=2}^{n}\left(a t_{-} l_{k}[j] \wedge \bigwedge_{i=2}^{n}\left((i \neq j) \Rightarrow \neg a t_{-} l_{k}[i]\right)\right)
$$

The set of compassion requirements $\left\{\left\langle 1, \neg a t_{-} l_{4}[i]\right\rangle \mid i=1, \ldots, n\right\}$ and the set $\left\{\left\langle 1, \neg a t_{-} l_{3}[i]\right\rangle \mid i=1, \ldots, n\right\}$ induce two new compassion requirements $F_{2}=$ $\left\langle 1, \neg \pi_{4}=1\right\rangle$ and $F_{3}=\left\langle 1, \neg \pi_{3}=1\right\rangle$. Then the set of compassion requirements of the abstract program is $\mathcal{F}=\left\{F_{1}, F_{2}, F_{3}\right\}$. 


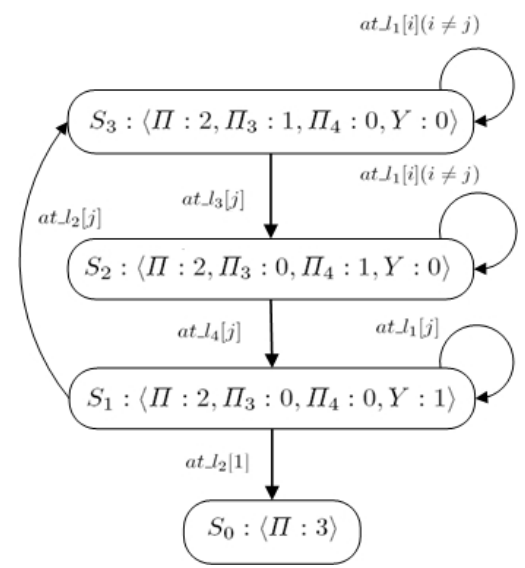

Fig. 4. The Pending Graph

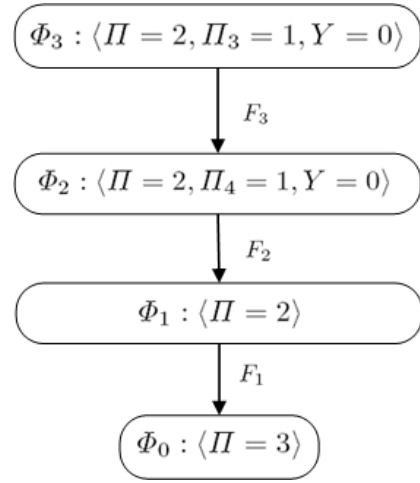

Fig. 5. The Abstract Graph

Let - denote any value in the range of the respective position of the abstract states. For instance, $(1,-,-,-)$ denote the abstract states where the value of $\left(\Pi, \Pi_{3}, \Pi_{4}, Y\right)$ satisfying $\Pi=1$ and the rest of the positions could be any value. Then the abstract states represented by the following tuples covers the reachable concrete states

$$
\begin{aligned}
& (1,-,-,-) \\
& (2,0,0,1) \\
& (2,1,0,0) \\
& (2,0,1,0) \\
& (3,-,-,-) \\
& (4,-,-,-)
\end{aligned}
$$

Let $S_{0}, S_{1}, S_{2}, S_{3}$ be the set of states represented by respectively

$$
(3,-,-,-),(2,0,0,1),(2,0,1,0),(2,1,0,0)
$$

Then we construct the pending graph (shown in shown in Fig. 4) with these four states with $S_{0}=g$. Let $R_{i}$ and $H_{i}$ be the rank of $S_{i}$ and the set of helpful compassion requirements associated to $S_{i}$, respectively, initially with $R_{i}=[]$ and $H_{i}=[]$. $[1]$.

We have two MSCCs: $\left\{S_{3}, S_{2}, S_{1}\right\}$ and $\left\{S_{0}\right\}$. Then $R_{0}=[0], R_{1}=R_{2}=R_{3}=$

By checking the MSCCs $\left\{S_{3}, S_{2}, S_{1}\right\}$ and $\left\{S_{0}\right\}$, we find that the first one violates the compassion requirement $F_{1}=\left\langle a t_{-} l_{2}[1] \wedge y=1\right.$, at_l $\left.l_{3}[1]\right\rangle$. Then $H_{1}=$ $H_{2}=H_{3}=\left[F_{1}\right]$.

We remove the edges $\left(S_{1}, S_{3}\right)$ and $\left(S_{1}, S_{1}\right)$, then apply C-RANK again. We obtain 3 MSCCs $\left\{S_{1}\right\},\left\{S_{2}\right\},\left\{S_{3}\right\}$. Then $R_{3}=[1,2], R_{2}=[1,1], R_{1}=[1,0]$.

By checking the 3 MSCCs, we find that $\left\{S_{2}\right\}$ violates $F_{2}=\left\langle 1, \neg \pi_{4}=1\right\rangle$ and $\left\{S_{3}\right\}$ violates $F_{3}=\left\langle 1, \neg \pi_{3}=1\right\rangle$. The compassion requirements are then added 
to the respective nodes, such that $H_{3}=\left[F_{1}, F_{3}\right], H_{2}=\left[F_{1}, F_{3}\right]$. The final value of $R_{i}$ and $H_{i}$ are as shown in Table 1.

\begin{tabular}{|c|l|l|l|}
\hline Index $i$ & $S_{i}$ & $R_{i}$ & $H_{i}$ \\
\hline 3 & $S_{3}$ & {$[1,2]$} & {$\left[F_{1}, F_{3}\right]$} \\
\hline 2 & $S_{2}$ & {$[1,1]$} & {$\left[F_{1}, F_{2}\right]$} \\
\hline 1 & $S_{1}$ & {$[1,0]$} & {$\left[F_{1}\right]$} \\
\hline 0 & $S_{0}$ & {$[0]$} & \\
\hline
\end{tabular}

Table 1. The Rank Table of Program MUX-SEM

According to $H_{i}$, we construct the abstract nodes by grouping together nodes that need satisfying the same compassion requirement. The abstract nodes of the program MUX-SEM with their respective ranks ${ }^{2}$ are listed in Table 2 and the abstract graph is shown in Fig. 5 . In the abstract graph, we mark " $F_{i}$ " as the label of edges to illuminate that the program must follow the transitions because of the compassion requirements. The transitions which do not relate to the compassion requirements are not shown in the abstract diagram.

\begin{tabular}{|c|c|c|c|}
\hline Abstract Node & Nodes & Rank & Compassion Req. \\
\hline$\Phi_{3}$ & $S_{3}$ & {$[1,2]$} & $F_{3}$ \\
\hline$\Phi_{2}$ & $S_{2}$ & {$[1,1]$} & $F_{2}$ \\
\hline$\Phi_{1}$ & $S_{1}, S_{2}, S_{3}$ & {$[1]$} & $F_{1}$ \\
\hline$\Phi_{0}$ & $S_{0}$ & {$[0]$} & \\
\hline
\end{tabular}

Table 2. The Abstract Table of Program MUX-SEM

Finally, we obtain the concrete helpful assertions $\varphi_{1}, \varphi_{2}, \varphi_{3}$ by concretizing the abstract assertions $\Phi_{1}, \Phi_{2}, \Phi_{3}$, and obtain the ranks $\Delta_{1}, \Delta_{2}, \Delta_{3}$ by renumbering the respective ranks in Fig. 5 . The concrete assertions $\varphi_{1}, \varphi_{2}, \varphi_{3}$ and the ranks $\Delta_{1}, \Delta_{2}, \Delta_{3}$ are shown in the table in Table 3.

Relating to the original program with $n$ processes, let $(3, j)$ denote the states where process $j$ is at label $l_{3}$ and $(4, j)$ denote the states where process $j$ is at label $l_{4}$ for $j=1, \ldots, n$, we obtain Table 4 .

The validity of the premises of rule C-RESPONSE for example 1 has been verified with the constructed auxiliary constructs $\varphi_{1}, \varphi_{2}, \varphi_{3}$ and $\Delta_{1}, \Delta_{2}, \Delta_{3}$. The reader is referred to Appendix A.1 for the details.

\footnotetext{
${ }^{2}$ Note that $F_{1}$ is associated to the nodes in the first level of computation of ranks, therefore the rank of $\Phi_{1}$ is only concerned with its first level projection.
} 


\begin{tabular}{|c|c|c|}
\hline Index $i$ & $\varphi_{i}$ & $\Delta_{i}$ \\
\hline 3 & $a t_{-} l_{2}[1] \wedge \bigvee_{j=2}^{n}\left(a t_{-} l_{3}[j] \wedge \bigwedge_{i=2}^{n}\left((i \neq j) \Rightarrow \neg a t_{-} l_{3}[i]\right)\right) \wedge y=0$ & {$[2]$} \\
\hline 2 & $a t_{-} l_{2}[1] \wedge \bigvee_{j=2}^{n}\left(a t_{-} l_{4}[j] \wedge \bigwedge_{i=2}^{n}\left((i \neq j) \Rightarrow \neg a t_{-} l_{4}[i]\right)\right) \wedge y=0$ & {$[1]$} \\
\hline 1 & $a t_{-} l_{2}[1]$ & {$[0]$} \\
\hline
\end{tabular}

Table 3. The Concrete Table of MUX-SEM Program

\begin{tabular}{|c|c|c|}
\hline index $i$ & $\varphi_{i}$ & $\Delta_{i}$ \\
\hline$(3, j), j>1$ & at_l $l_{2}[1] \wedge a t_{-} l_{3}[j] \wedge y=0$ & {$[2]$} \\
\hline$(4, j), j>1$ & $a t_{-} l_{2}[1] \wedge a t_{-} l_{4}[j] \wedge y=0$ & {$[1]$} \\
\hline$(-, 1)$ & $a t_{-} l_{2}[1]$ & {$[0]$} \\
\hline
\end{tabular}

Table 4. The Concrete Table of MUX-SEM Program

\subsection{Example 2: COND-TERM}

Fig. 6 shows the program COND-TERM. The response property we wish to establish is $\Psi: a t_{-} l_{1} \Rightarrow \diamond a t_{-} l_{4}$. The just requirements are $\neg a t_{-} l_{i}$ for $i=1,2,3,4$, and the compassion requirements is $F_{1}=\left\langle a t_{-} l_{3} \wedge x=0,0\right\rangle$. Let $F_{i+1}$ be $\left\langle 1, \neg a t_{-} l_{i}\right\rangle$ representing the just requirements for $i=1,2,3$.

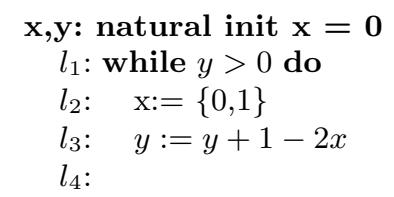

Fig. 6. Program COND-TERM

Let $d e c_{y}=\operatorname{sign}\left(y-y^{\prime}\right)$ in which $y$ denotes the value of $y$ in the previous state and $y^{\prime}$ denotes the value of $y$ in the current state. The abstraction mapping $\alpha$ is defined by :

$$
\alpha: \Pi=\pi, X=(x>0), Y=(y>0), D e c_{y}=d e c_{y}
$$

where $\Pi=i$ denotes $a t_{-} l_{i}$ is true. We construct the pending graph as showing in Fig. 7. The constraints of the graph include the additional compassion requirement $F_{D}=\left\langle D e c_{y}>0, D e c_{y}<0\right\rangle$ which is deduced from the condition of the while loop according to the rank abstraction process.

There are 8 states $\left\{S_{0}, S_{1}, \ldots, S_{7}\right\}$ with $S_{0}=g$. Let $R_{i}$ and $H_{i}$ be the rank of $S_{i}$ and the set of helpful compassion requirements associated to $S_{i}$, respectively, initially with $R_{i}=$ [] and $H_{i}=$ [].

In the first level of computation, we have 4 MSCCs: $\{g\},\left\{S_{1}\right\},\left\{S_{2}, S_{3}, S_{4}, S_{5}\right.$, $\left.S_{6}\right\},\left\{S_{7}\right\}$. Then $R_{0}=[0], R_{1}=[1], R_{2}=R_{3}=R_{4}=R_{5}=R_{6}=[2], R_{7}=[3]$. 


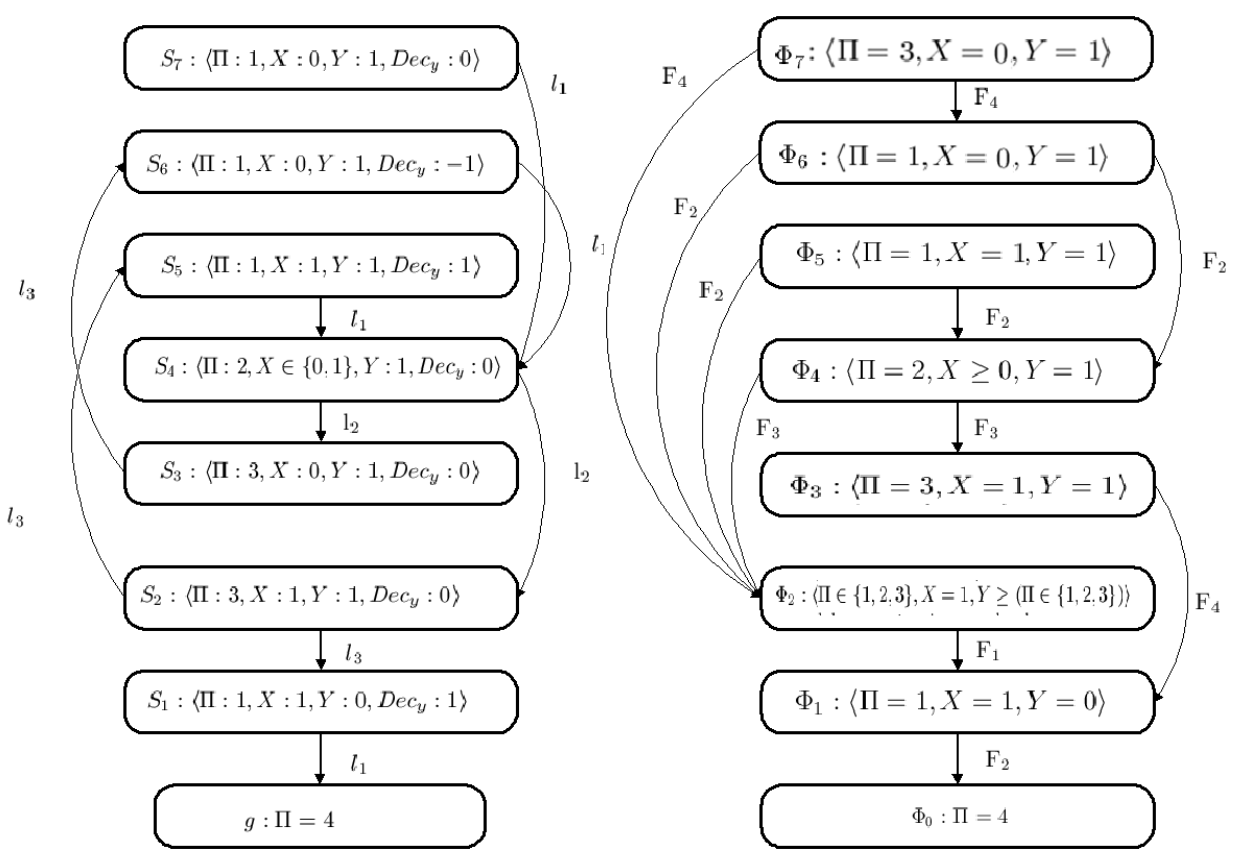

Fig. 7. The Pending Graph

Fig. 8. The Abstract Graph

Let $C_{0}, C_{1}, C_{2}, C_{3}$ denote the 4 MSCCs. By checking the MSCCs (excluding $C_{0}$ ) against the compassion requirements, we obtain the table as shown in Table 5.

\begin{tabular}{|c|c|}
\hline Component & Violation \\
\hline$C_{1}$ & $F_{2}$ \\
\hline$C_{2}$ & $F_{1}$ \\
\hline$C_{3}$ & $F_{2}$ \\
\hline
\end{tabular}

Table 5. The MSCCs $C_{1}, C_{2}, C_{3}$

\begin{tabular}{|c|c|}
\hline Component & Violation \\
\hline$C_{21}$ & $F_{4}$ \\
\hline$C_{22}$ & $F_{2}$ \\
\hline$C_{23}$ & $F_{D}$ \\
\hline
\end{tabular}

Table 6. The MSCCs $C_{21}, C_{22}, C_{23}$

Then we add the respective compassion requirement to $H_{1}, \ldots, H_{7}$ such that $H_{1}=H_{7}=\left[F_{2}\right], H_{2}=H_{3}=H_{4}=H_{5}=H_{6}=\left[F_{1}\right]$.

Since $C_{2}$ is not a non-trivial subgraph, we remove the edge $\left(S_{4} \rightarrow S_{3}\right)$, which leads into the state satisfying at $l_{3} \wedge x=0$, from $C_{2}$, and compute again with the modified subgraph.

In the second level of computation, we have 3 MSCCs: $\left\{S_{3}\right\},\left\{S_{6}\right\},\left\{S_{4}, S_{2}\right.$, $\left.S_{5}\right\}$. Then $R_{3}=[2,2], R_{6}=[2,1], R_{2}=R_{4}=R_{5}=[2,0]$. 
Let $C_{21}, C_{22}, C_{23}$ denote the 3 MSCCs. By checking the MSCCs against the compassion requirements, we obtain the table as shown in Table 6.

Then we add the respective compassion requirement to $H_{2}, \ldots, H_{6}$. In addition, since $C_{23}=\left\{S_{4}, S_{2}, S_{5}\right\}$ violates $F_{D}=\left\langle D e c_{y}>0, D e c_{y}<0\right\rangle$, we add $y$ to $R_{2}, R_{4}, R_{5}$. Then since $C_{23}$ is not a non-trivial subgraph, we remove the edge $\left(S_{2} \rightarrow S_{5}\right)$, which leads into the state satisfying $D e c_{y}>0$, from $C_{23}$, and compute again with the modified subgraph.

In the third level of computation, we have 3 MSCCs: $\left\{S_{5}\right\},\left\{S_{4}\right\},\left\{S_{2}\right\}$. The rank to be assigned to the nodes in this level is $2,1,0$.

Then $R_{5}=[2,1, y, 2], R_{4}=[2,1, y, 1], R_{2}=[2,1, y, 0]$. Since $\left\{S_{5}\right\},\left\{S_{4}\right\}$ and $\left\{S_{2}\right\}$ are trivial MSCCs, we add $F_{2}, F_{3}, F_{4}$ to $H_{5}, H_{4}, H_{2}$ respectively. The final value of $R_{i}$ and $H_{i}$ are as shown in Table 7 .

\begin{tabular}{|c|l|l|l|}
\hline Index $i$ & $S_{i}$ & $R_{i}$ & $H_{i}$ \\
\hline 7 & $S_{7}$ & {$[3]$} & {$\left[F_{2}\right]$} \\
\hline 6 & $S_{6}$ & {$[2,1]$} & {$\left[F_{1}, F_{2}\right]$} \\
\hline 5 & $S_{5}$ & {$[2,0, y, 2]$} & {$\left[F_{1}, F_{D}, F_{2}\right]$} \\
\hline 4 & $S_{4}[2,0, y, 1]$ & {$\left[F_{1}, F_{D}, F_{3}\right]$} \\
\hline 3 & $S_{3}$ & {$[2,2]$} & {$\left[F_{1}, F_{4}\right]$} \\
\hline 2 & $S_{2}$ & {$[2,0, y, 0]$} & {$\left[F_{1}, F_{D}, F_{4}\right]$} \\
\hline 1 & $S_{1}$ & {$[1]$} & {$\left[F_{2}\right]$} \\
\hline 0 & $S_{0}$ & {$[0]$} & \\
\hline
\end{tabular}

Table 7. The Rank Table of Program COND-TERM

According to $H_{i}$, we construct the abstract nodes ${ }^{3}$ by grouping together nodes that need satisfying the same compassion requirement merging $S_{6}-S_{2}$ as an abstract state $\Phi_{2}$ and by grouping together nodes that agree with the value of all variables except Dec: merging $S_{7}$ and $S_{5}$ as another abstract state $\Phi_{6}$.

The abstract nodes with their respective ranks are listed in Table 8 and the abstract graph is shown in Fig. 8.

Finally, we obtain the concrete helpful assertions $\varphi_{1}, \ldots, \varphi_{7}$ by concretizing the abstract assertions $\Phi_{1}, \ldots, \Phi_{7}$, and obtain the ranks $\Delta_{1}, \ldots, \Delta_{7}$ by renumbering the respective ranks in Fig. 8 . The helpful assertions and the ranks are shown in the table in Table 9.

The validity of the premises of rule C-RESPONSE for example 2 has been verified with the constructed auxiliary constructs $\varphi_{1}, \ldots, \varphi_{7}$ and $\Delta_{1}, \ldots, \Delta_{7}$. The reader is referred to Appendix A.2 for the details.

\footnotetext{
${ }^{3}$ Note that $F_{D}$ is not involved in the construction of abstract nodes, since it is not one of the original system constraints.
} 


\begin{tabular}{|c|c|c|c|}
\hline Abstract Node & Nodes & Rank & Compassion Req. \\
\hline$\Phi_{7}$ & $S_{3}$ & {$[2,2]$} & $F_{4}$ \\
\hline$\Phi_{6}$ & $S_{6}, S_{7}$ & {$[2,1]$} & $F_{2}$ \\
\hline$\Phi_{5}$ & $S_{5}$ & {$[2,0, y, 2]$} & $F_{2}$ \\
\hline$\Phi_{4}$ & $S_{4}$ & {$[2,0, y, 1]$} & $F_{3}$ \\
\hline$\Phi_{3}$ & $S_{2}$ & {$[2,0, y, 0]$} & $F_{4}$ \\
\hline$\Phi_{2}$ & $S_{2}, \ldots, S_{6}$ & {$[2]$} & $F_{1}$ \\
\hline$\Phi_{1}$ & $S_{1}$ & {$[1]$} & $F_{2}$ \\
\hline$\Phi_{0}$ & $S_{0}$ & {$[0]$} & \\
\hline
\end{tabular}

Table 8. The Abstract Table of Program COND-TERM

\begin{tabular}{|c|c|c|}
\hline Index & $\varphi_{i}$ & $\Delta_{i}$ \\
\hline 7 & $a t_{-} l_{3} \wedge y>0 \wedge x=0$ & {$[2,2]$} \\
\hline 6 & $a t_{-} l_{1} \wedge y>0 \wedge x=0$ & {$[2,1]$} \\
\hline 5 & $a t_{-} l_{1} \wedge y>0 \wedge x=1$ & {$[2,0, y, 2]$} \\
\hline 4 & $a t_{-} l_{2} \wedge y>0 \wedge x \in\{0,1\}$ & {$[2,0, y, 1]$} \\
\hline 3 & $a t_{-} l_{3} \wedge y>0 \wedge x=1$ & {$[2,0, y, 0]$} \\
\hline 2 & at_l $l_{1 . .3} \wedge y \geqslant a t_{-} l_{1,2,3} \wedge x \in\{0,1\}$ & {$[2]$} \\
\hline 1 & $a t_{-} l_{1} \wedge y=0 \wedge x=1$ & [1] \\
\hline
\end{tabular}

Table 9. The Concrete Table of Program COND-TERM

\subsection{Example 3: UP-DOWN}

Fig. 9 shows the program UP-DOWN. This program can be handled by RANKJUST-GRAPH [2]. Here we illustrate how it is handled by C-GRAPH, in order to get an impression of the similarity and differences of these two approaches. The abstract program is shown in Fig.10.

The response property is $\left(\pi_{1}=0 \wedge \pi_{2}=0\right) \Rightarrow \diamond\left(\pi_{1}=4\right)$, where $\pi_{1}$ and $\pi_{2}$ are the location counters for $P_{1}$ and $P_{2}$. They are denoted by $l_{i}$ and $m_{i}$, respectively.

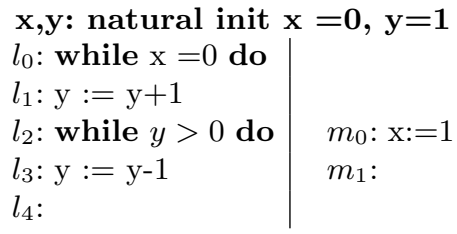

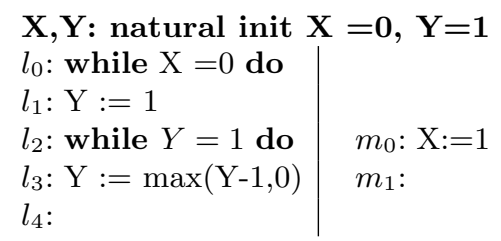

Fig. 10. Abstract Program UP-DOWN

Fig. 9. Program UP-DOWN

We also use the notation $a t_{-} l_{i}$ to denote $\pi_{1}=i$, and at_m $m_{j}$ to denote $\pi_{2}=j$. The justice requirements of the program include $F_{0}:\left\langle 1, \neg a t l_{0}\right\rangle, F_{1}:\left\langle 1, \neg a t_{-} l_{1}\right\rangle$, $F_{2}:\left\langle 1, \neg a t_{-} l_{2}\right\rangle, F_{3}:\left\langle 1, \neg a t_{-} l_{3}\right\rangle, F_{4}:\left\langle 1, \neg a t_{-} m_{1}\right\rangle$. Employing the predicate base 
$\mathcal{P}: x>0, y>0$, we obtain the abstraction

$$
\alpha: \Pi_{1}=\pi_{1}, \Pi_{2}=\pi_{2}, X=(x>0), Y=(y>0)
$$

The abstract property is $\Psi_{\alpha}:\left(\Pi_{1}=0 \wedge \Pi_{2}=0\right) \Rightarrow \diamond\left(\Pi_{1}=4\right)$.

The pending graph is shown in Fig.11 and the abstract diagram is shown in Fig.12.

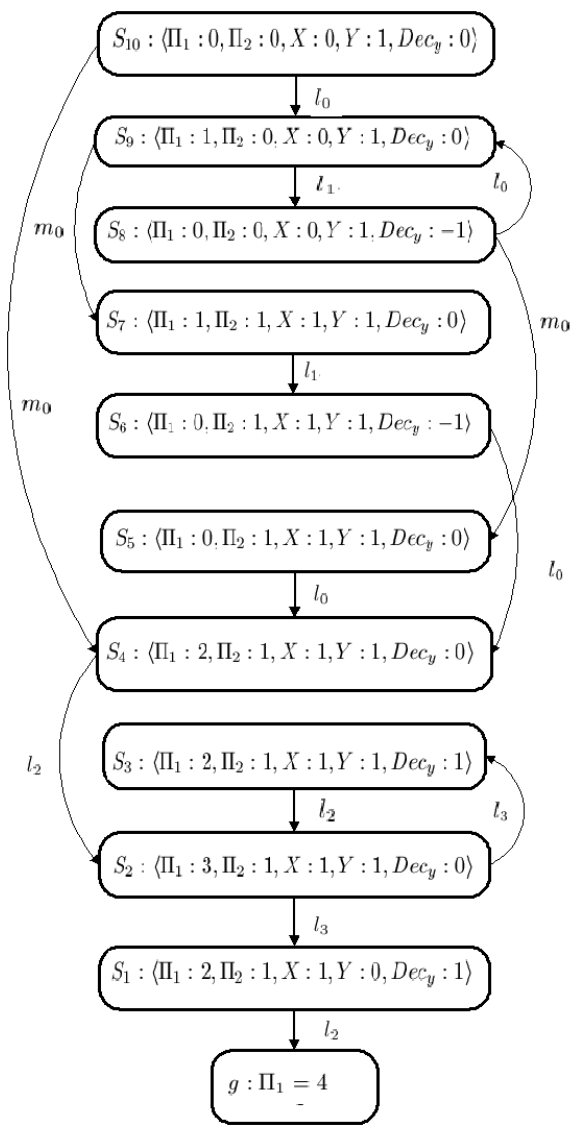

Fig. 11. The Pending Graph

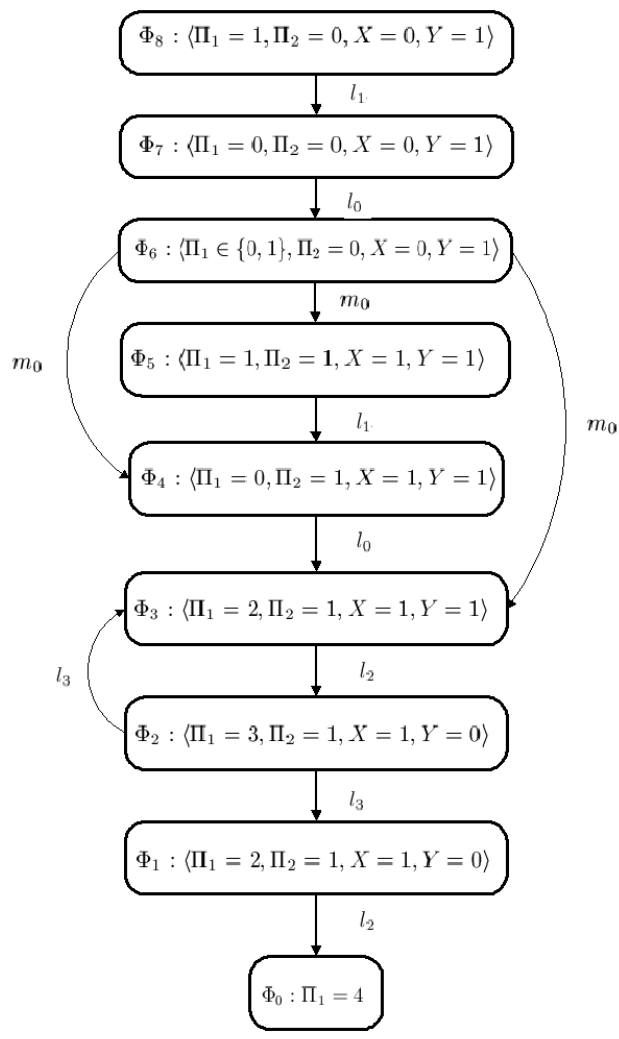

Fig. 12. The Abstract Graph

In the first level of computation, the MSCC decomposition yields the following sorted list: g $, S_{1},\left\{S_{2}, S_{3}\right\}, S_{4}, S_{5}, S_{6}, S_{7},\left\{S_{8}, S_{9}\right\}, S_{10}$. Consequently, we assign to nodes $\mathrm{g}, S_{1}, \ldots, S_{7}$ the sequence of ranks: $0,1,2,2,3,4,5,6,7,7,8$.

In the second level of computation, we consider the MSCCs $\left\{S_{2}, S_{3}\right\}$ and $\left\{S_{8}, S_{9}\right\}$. Table 10 shows the final values of $R_{i}$ and $H_{i}$. 


\begin{tabular}{|c|l|l|l|}
\hline Index $i$ & $S_{i}$ & $R_{i}$ & $H_{i}$ \\
\hline 10 & $S_{10}$ & {$[8]$} & {$\left[F_{0}, F_{5}\right]$} \\
\hline 9 & $S_{9}$ & {$[7,1]$} & {$\left[F_{5}, F_{1}\right]$} \\
\hline 8 & $S_{8}$ & {$[7,0]$} & {$\left[F_{5}, F_{0}\right]$} \\
\hline 7 & $S_{7}$ & {$[6]$} & {$\left[F_{1}\right]$} \\
\hline 6 & $S_{6}$ & {$[5]$} & {$\left[F_{0}\right]$} \\
\hline 5 & $S_{5}$ & {$[4]$} & {$\left[F_{0}\right]$} \\
\hline 4 & $S_{4}$ & {$[3]$} & {$\left[F_{2}\right]$} \\
\hline 3 & $S_{3}$ & {$[2, y, 1]$} & {$\left[F_{2}, F_{D}\right]$} \\
\hline 2 & $S_{2}$ & $[2, y, 0)]$ & {$\left[F_{3}, F_{D}\right]$} \\
\hline 1 & $S_{1}$ & {$[1]$} & {$\left[F_{2}\right]$} \\
\hline 0 & $S_{0}$ & {$[0]$} & \\
\hline
\end{tabular}

Table 10. The Rank Table of Program UP-DOWN

According to $R_{i}$ and $H_{i}$ for $i=1, \ldots, 8$, we construct abstract states with their associated ranks as shown in Table 11. The the abstract graph is shown in Fig.12.

\begin{tabular}{|c|c|c|c|}
\hline Abstract Node & Nodes & Rank & Compassion Req. \\
\hline$\Phi_{8}$ & $S_{9}$ & {$[7]$} & $F_{1}$ \\
\hline$\Phi_{7}$ & $S_{8}, S_{10}$ & {$[6]$} & $F_{0}$ \\
\hline$\Phi_{6}$ & $S_{9}, S_{8}, S_{10}$ & {$[5]$} & $F_{5}$ \\
\hline$\Phi_{5}$ & $S_{7}$ & {$[4]$} & $F_{1}$ \\
\hline$\Phi_{4}$ & $S_{5}, S_{6}$ & {$[3]$} & $F_{0}$ \\
\hline$\Phi_{3}$ & $S_{3}, S_{4}$ & {$[2, y, 1]$} & $F_{2}$ \\
\hline$\Phi_{2}$ & $S_{2}$ & {$[2, y, 0]$} & $F_{3}$ \\
\hline$\Phi_{1}$ & $S_{1}$ & {$[1]$} & $F_{2}$ \\
\hline$\Phi_{0}$ & $S_{0}$ & {$[0]$} & \\
\hline
\end{tabular}

Table 11. The Abstract Table of Program UP-DOWN

At the last step, we compute the concrete helpful assertions $\varphi_{1}, \ldots, \varphi_{8}$ by concretization of the abstract assertions $\Phi_{1}, \ldots, \Phi_{8}$. The concrete helpful assertions are shown in the table in Table 12.

The validity of the premises of rule C-RESPONSE for example 3 has been verified with the constructed auxiliary constructs $\varphi_{1}, \ldots, \varphi_{8}$ and $\Delta_{1}, \ldots, \Delta_{8}$. The reader is referred to Appendix A.3 for the details.

\subsection{Discussion}

On different compassion requirements Suppose that in the first example, we replace the original compassion requirement $\left\langle a t_{-} l_{2}[1] \wedge y, a t_{-} l_{3}[1]\right\rangle$ by $\left\langle a t_{-} l_{2}[1] \wedge\right.$ 


\begin{tabular}{|c|c|c|}
\hline Index & $\varphi_{i}$ & $\Delta_{i}$ \\
\hline 8 & $a t_{-} l_{1} \wedge a t \_m_{0} \wedge x=0 \wedge y>0$ & {$[7]$} \\
\hline 7 & $a t_{-} l_{0} \wedge a t_{-} m_{0} \wedge x=0 \wedge y>0$ & [6] \\
\hline 6 & at_l $l_{0,1} \wedge a t \_m_{0} \wedge x=0 \wedge y>0$ & [5] \\
\hline 5 & $a t_{-} l_{1} \wedge a t_{-} m_{1} \wedge x>0 \wedge y>0$ & [4] \\
\hline 4 & $a t \_l_{0} \wedge a t \_m_{1} \wedge x>0 \wedge y>0$ & {$[3]$} \\
\hline 3 & $a t_{-} l_{2} \wedge a t_{-} m_{1} \wedge x>0 \wedge y>0$ & {$[2, y, 1]$} \\
\hline 2 & $a t_{-} l_{3} \wedge a t_{-} m_{1} \wedge x>0 \wedge y>0$ & {$[2, y, 0]$} \\
\hline 1 & $a t_{-} l_{2} a t_{-} m_{1} \wedge x>0 \wedge y=0$ & [1] \\
\hline
\end{tabular}

Table 12. The Concrete Table of Program UP-DOWN

$\left.y \wedge a t_{-} l_{2}[j], a t_{-} l_{3}[1]\right\rangle$. According to the approach, we can also obtain a set of auxiliary assertions and ranks, however these are too weak for the deductive proof of the liveness property. Since the modified system does not satisfy the liveness property, because the fairness requirement is not strong enough, the result is as expected. Details are in Appendix A.1 (Example 1a).

On the construction of pending graph We may construct a pending graph by grouping states together to obtain a smaller graph. However this may cause problems, if it is not done carefully. Suppose that in the second example, we construct the pending graph as shown in Fig. 13, which is more compact than that used in the second example. The problem with this pending graph is explained

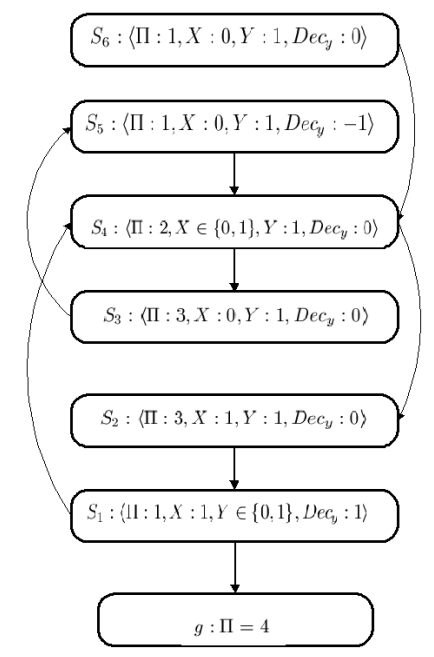

Fig. 13. Another Pending Graph of COND-TERM 
as follows. Since the MSCC $\left\{S_{4}, S_{2}, S_{1}\right\}$ violates $F_{D}:\left\langle D e c_{y}>0, D e c_{y}<0\right\rangle$, we have to remove some edges of the MSCC in order to continue the further analysis. If we remove the edge $\left(S_{2} \rightarrow S_{1}\right)$, then the rank of $S_{2}$ is the minimum. However it does not necessarily make progress to the goal state, because the value of variable $\mathrm{X}$ in $S_{1}$ includes 1 and 0 . Therefore we have to separate the state $\left\langle\Pi: 1, X \in\{0,1\}, Y: 0, D e c_{y}: 1\right\rangle$ into two different states: $\left\langle\Pi: 1, X: 0, Y: 0, D_{e c}: 1\right\rangle$ and $\left\langle\Pi: 1, X: 1, Y: 0, D e c_{y}: 1\right\rangle$. This modification makes the pending graph of COND-TERM that we used in Section 4.2 .

\section{$5 \quad$ Concluding Remarks}

For proving the response property in systems with fairness based on the rule presented in [13], we need auxiliary constructs. We have presented a method for extracting such constructs. The method extends that presented in [2] which aimed at proving the response property in systems with justice. When the system is restricted to only allow justice requirements, the auxiliary constructs we

obtained may be different from that obtained by using the method presented in [2].

\section{References}

1. Arons T, Pnueli A, Ruah S, Xu Y, Zuck L D. Parameterized Verification with Automatically Computed Inductive Assertions. Proc. Computer Aided Verification, volume 2102 of LNCS, pp.221-234. Springer,2001.

2. Balaban I, Pnueli A, Zuck L D. Modular ranking abstraction. International Journal of Foundations of Computer Science, 2007, 18(1): 5-44.

3. Balaban I, Pnueli A, Zuck L D. Ranking abstraction as companion to predicate abstraction. In Proc. FORTE, Taipei, Taiwan, October 2-5, 2005, pp.1-12.

4. Ball T, Majumdar R, Millstein T, Rajamani S. Automatic predicate abstraction if C programs. Proc. PLDI, volume 36 of ACM SIGPLAN Notices, pp.203-213. ACM Press, 2001.

5. Das S. Predicate Abstraction. Stanford University, 2003.

6. Fang Y, Piterman N, Pnueli A, Zuck L D. Liveness by invisible ranking. Proc. VMCAI2004, LNCS 2937, pp.223-238, 2004.

7. Fang Y, Piterman N, Pnueli A, Zuck L D. Liveness with incomprehensible ranking. In Proc. Tools and Algorithms for the Construction and Analysis of Systems, Barcelona, Spain, Mar. 29-Apr. 2, 2004, pp.482-496.

8. Graf S, Saidi H. Construction of abstract state graph with PVS. Proc. Computer Aided Verification, volume 1254 of LNCS, pp.72-83. Springer,1997.

9. Kesten Y, Pnueli A. Verification by augmented finitary abstraction. Information and Computation, 2000, 163(1): 203-243.

10. Kesten Y, Pnueli A, Vardi M. Verification by augmented abstraction: The automata theoretic view. J.Comp $\dot{z}$ systems Sci, 2001, 62:668-690.

11. Manna Z, Pnueli A. Completing the temporal picture. TCS, 1991, 83(1):91-130.

12. Manna z, Pnueli A. Temporal verification diagrams. Theoretical Aspects of Computer Software, Volume 789:726-765,1994.

13. Pnueli A, Sa'ar Y. All you need is compassion. In Proc. VMCAI, San Francisco, USA, January 7-9, 2008, pp.33-247. 


\section{A Appendix}

This appendix contains the steps of checking whether the premisses of the rule C-RESPONSE is valid by utilizing the auxiliary constructs produced by CGRAPH.

\section{A.1 Example 1: MUX-SEM}

The goal represented by $p \rightarrow \diamond q$, the compassion requirements represented by $\left(p_{i}, q_{i}\right)$, and the auxiliary constructs for example 1 are listed as follows.

\begin{tabular}{|l|l|l|l|}
\hline p: $a t_{-} l_{2}[1]$ & q: $a t_{-} l_{3}[1]$ & & \\
\hline$\varphi_{1}: a t_{-} l_{2}[1]$ & $p_{1}: a t_{-} l_{2}[1] \wedge y=1$ & $q_{1}: a t_{-} l_{3}[1]$ & $\Delta_{1}:[0]$ \\
\hline$\varphi_{2}: a t_{-} l_{2}[1] \wedge a t_{-} l_{4}[j] \wedge y=0$ & $p_{2}: 1$ & $q_{2}: \neg a t_{-} l_{4}[j]$ & $\Delta_{2}:[1]$ \\
\hline$\varphi_{3}: a t_{-} l_{2}[1] \wedge a t_{-} l_{3}[j] \wedge y=0$ & $p_{3}: 1$ & $q_{3}: \neg a t_{-} l_{3}[j]$ & $\Delta_{3}:[2]$ \\
\hline
\end{tabular}

We prove that the premisses of the rule C-RESPONSE hold when using the above auxiliary constructs as follows. In the proof, $S_{i}$ which represents a subset of the concrete states is the corresponding state in Fig. 4.

$$
\begin{aligned}
\mathrm{R} 1 \mathrm{p} \Rightarrow \mathrm{q} \vee & \bigvee_{j=1}^{n}\left(p_{j} \wedge \varphi_{j}\right) \\
S_{3}, S_{2}, S_{1} & p_{1} \wedge \varphi_{1}: S_{1} \\
& p_{2} \wedge \varphi_{2}: S_{2} \\
& p_{3} \wedge \varphi_{3}: S_{3}
\end{aligned}
$$

This means that the initial states $S_{3}, S_{2}, S_{1}$ which satisfy $p:$ at_l $l_{2}[1]$ can be covered by the set of states specified on the right hand side. This proof uses information provided by the pend graph. Formally, the proof of $p \Rightarrow q \vee \bigvee_{j=1}^{n}\left(p_{j} \wedge\right.$ $\left.\varphi_{j}\right)$ may be carried out (without using the pend graph) as follows.

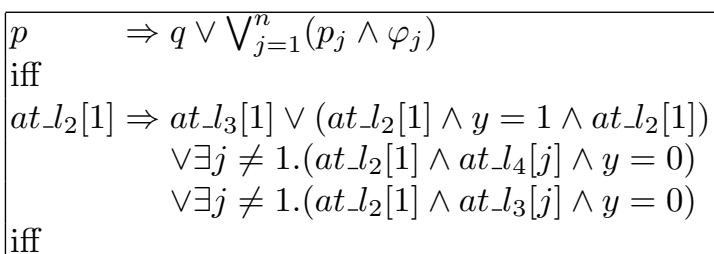

$$
\begin{aligned}
& a t_{-} l_{2}[1] \Rightarrow a t_{-} l_{3}[1] \vee(y=1) \\
& \vee \exists j \neq 1 .\left(a t_{-} l_{4}[j] \wedge y=0\right) \\
& \vee \exists j \neq 1 .\left(a t_{-} l_{3}[j] \wedge y=0\right) \\
& \text { iff } \\
& \text { at_l } l_{2}[1] \Rightarrow(y=1) \\
& \vee \exists j \neq 1 .\left(a t_{-} l_{4}[j] \wedge y=0\right) \\
& \vee \exists j \neq 1 .\left(a t_{-} l_{3}[j] \wedge y=0\right) \\
& \text { only if } \\
& y \neq 1 \quad \Rightarrow \exists j .\left(a t_{-} l_{4}[j]\right) \vee \exists j .\left(a t_{-} l_{3}[j]\right)
\end{aligned}
$$

The last implication holds, because it is an invariant of the program. In the following, for simplicity, we use the pend graph as the basis of our proofs of the validity of R2-R4, and omit this kind of details. 


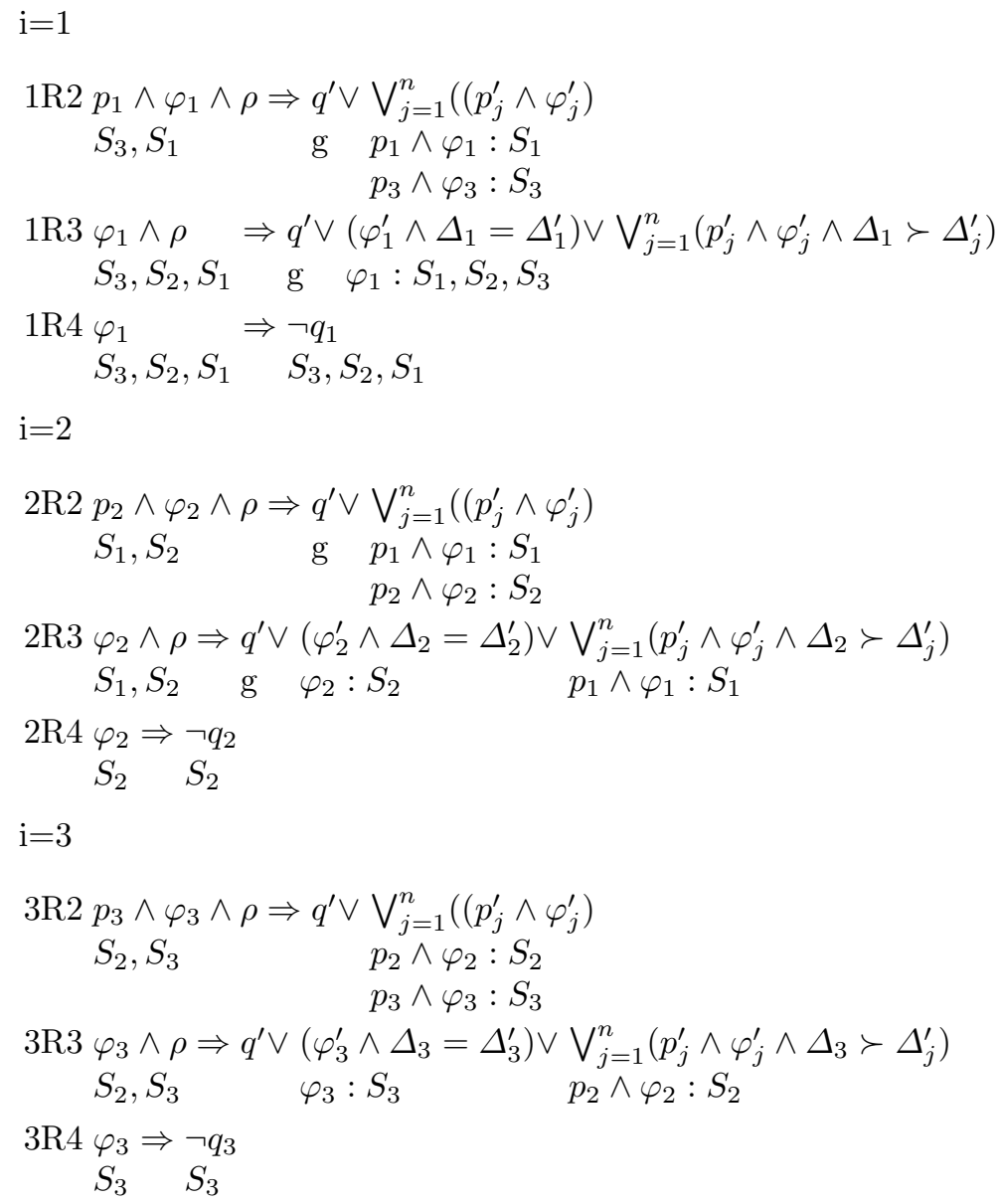

This means that the transitions from $\varphi_{i}$ and $p_{i} \wedge \varphi_{i}$ satisfy $R_{2}-R_{4}$. Therefore we can reach the goal state: $a t_{-} l_{3}[1]$ with these transitions, and the liveness property holds.

For illustration, the transitions of the program with 3 processes are shown in Fig. 14- Fig. 17 . The labels on the transitions indicate how states are changed according to the premisses of the rule, such that if we start from some state satisfying the initial assertion $p$, then we have transitions satisfy the premisses of the rule, and we can reach the goal state with these transitions. 


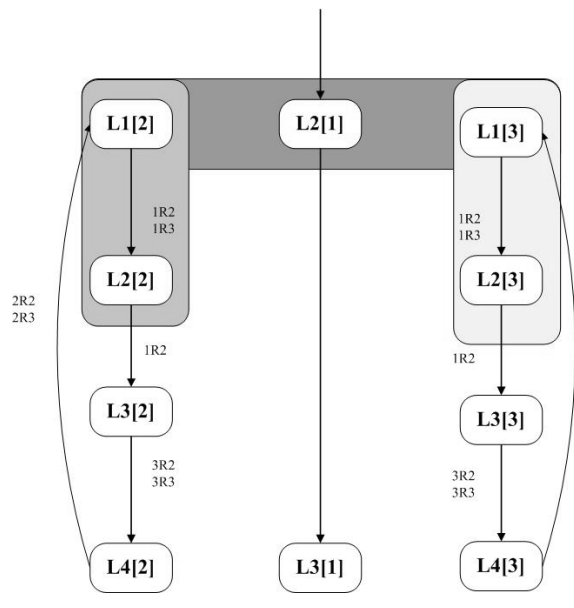

Fig. 14. $S_{1}$ in 3-Processes MUX

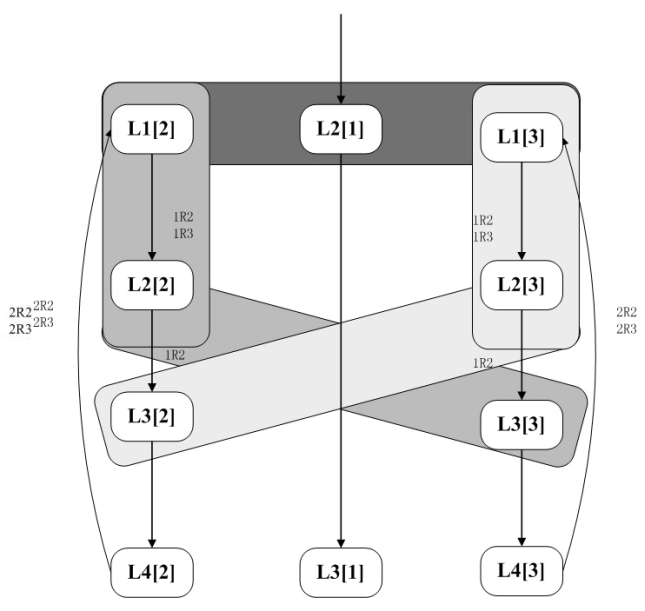

Fig. 15. $S_{2}$ in 3-Processes MUX

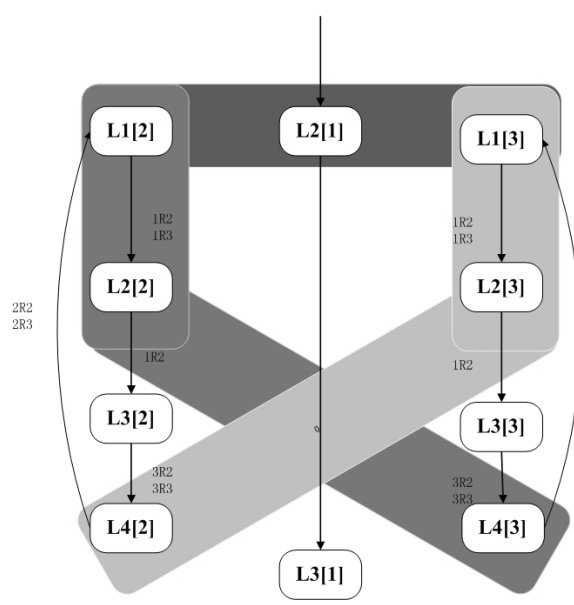

Fig. 16. $S_{3}$ in 3-Processes MUX

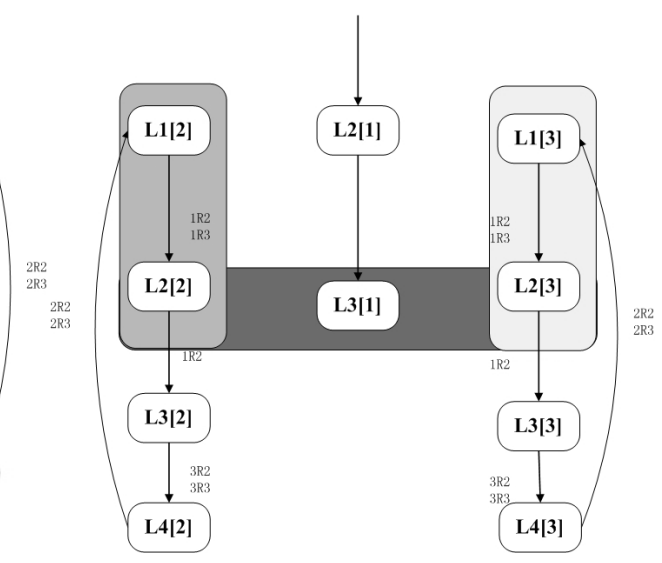

Fig. 17. $S_{0}$ in 3 -Processes MUX 


\section{A.2 Example 2: COND-TERM}

The goal, the compassion requirements and the auxiliary constructs for example 2 are listed as follows.

\begin{tabular}{|c|c|c|c|}
\hline p: $a t_{-} l_{1}$ & q: $a t_{-} l_{3}$ & & \\
\hline$\varphi_{1}: a t_{-} l_{1} \wedge y=0$ & $p_{1}: 1$ & $q_{1}: \neg a t_{-} l_{1}$ & $\Delta_{1}:[1]$ \\
\hline$\varphi_{2}: a t_{-} l_{1 . .3} \wedge y \geqslant a t_{-} l_{1,2,3} \wedge x \in\{0,1\}$ & $p_{2}: a t_{-} l_{3} \wedge x=0$ & $q_{2}: 0$ & $\Delta_{2}:[2]$ \\
\hline$\varphi_{3}: a t_{-} l_{3} \wedge y>0 \wedge x=1$ & $p_{3}: 1$ & $q_{3}: \neg a t_{-} l_{3}$ & $\Delta_{3}:[2,0, \mathrm{y}, 0]$ \\
\hline$\varphi_{4}: a t_{-} l_{2} \wedge y>0 \wedge x \in\{0,1\}$ & $p_{4}: 1$ & $q_{4}: \neg a t_{-} l_{2}$ & $\Delta_{4}:[2,0, \mathrm{y}, 1]$ \\
\hline$\varphi_{5}: a t_{-} l_{1} \wedge y>0 \wedge x=1$ & $p_{5}: 1$ & $q_{5}: \neg a t_{-} l_{1}$ & $\Delta_{5}:[2,0, \mathrm{y}, 2]$ \\
\hline$\varphi_{6}: a t_{-} l_{1} \wedge y>0 \wedge x=0$ & $p_{6}: 1$ & $q_{6}: \neg a t_{-} l_{1}$ & $\Delta_{6}:[2,1]$ \\
\hline$\varphi_{7}: a t_{-} l_{3} \wedge y>0 \wedge x=0$ & $p_{7}: 1$ & $q_{7}: \neg a t_{-} l_{3}$ & $\Delta_{7}:[2,2]$ \\
\hline
\end{tabular}

We prove that the premisses of the rule C-RESPONSE hold when using the above auxiliary constructs as follows.

$$
\begin{aligned}
\mathrm{R} 1 \mathrm{p}_{S_{7}, S_{6}, S_{5}, S_{1}}^{\Rightarrow \mathrm{q}} \vee & \vee \bigvee_{j=1}^{n}\left(p_{j} \wedge \varphi_{j}\right) \\
& p_{1} \wedge \varphi_{1}: S_{1} \\
& p_{5} \wedge \varphi_{5}: S_{5} \\
& p_{6} \wedge \varphi_{6}: S_{6}, S_{7}
\end{aligned}
$$

This means that the initial states $S_{7}, S_{6}, S_{5}, S_{1}$ which satisfy $p: a t_{-} l_{1}$ can be covered by the set of states specified on the right hand side.

$$
\begin{aligned}
& \mathrm{i}=1 \\
& 1 \mathrm{R} 2 p_{1} \wedge \varphi_{1} \wedge \rho \Rightarrow q^{\prime} \vee \bigvee_{j=1}^{n}\left(\left(p_{j}^{\prime} \wedge \varphi_{j}^{\prime}\right)\right. \\
& \mathrm{g} \quad \mathrm{g} \\
& 1 \mathrm{R} 3 \varphi_{1} \wedge \rho \Rightarrow q^{\prime} \vee\left(\varphi_{1} \wedge \Delta_{1}=\Delta_{1}^{\prime}\right) \vee \bigvee_{j=1}^{n}\left(p_{j}^{\prime} \wedge \varphi_{j}^{\prime} \wedge \Delta_{1} \succ \Delta_{j}^{\prime}\right) \\
& \mathrm{g} \quad \mathrm{g} \\
& \operatorname{1R} 4 \varphi_{1} \Rightarrow \neg q_{1} \\
& S_{1} \quad S_{1} \\
& \mathrm{i}=2 \\
& 2 \mathrm{R} 2 p_{2} \wedge \varphi_{2} \wedge \rho \Rightarrow q^{\prime} \vee \bigvee_{j=1}^{n}\left(\left(p_{j}^{\prime} \wedge \varphi_{j}^{\prime}\right)\right. \\
& S_{6} \quad p_{6} \wedge \varphi_{6}: S_{6} \\
& 2 \mathrm{R} 3 \varphi_{2} \wedge \rho \quad \Rightarrow q^{\prime} \vee\left(\varphi_{2}^{\prime} \wedge \Delta_{2}=\Delta_{2}^{\prime}\right) \vee \quad \bigvee_{j=1}^{n}\left(p_{j}^{\prime} \wedge \varphi_{j}^{\prime} \wedge \Delta_{2} \succ \Delta_{j}^{\prime}\right) \\
& S_{6}, S_{5}, S_{4}, S_{3}, S_{2}, S_{1} \quad \mathrm{~g} \quad \varphi_{2}: S_{6}, S_{5}, S_{4}, S_{3}, S_{2} p_{1} \wedge \varphi_{1}: S_{1}
\end{aligned}
$$

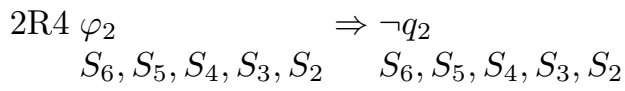

$$
\begin{aligned}
& \mathrm{i}=3 \\
& 3 \mathrm{R} 2 p_{3} \wedge \varphi_{3} \wedge \rho \Rightarrow q^{\prime} \vee \bigvee_{j=1}^{n}\left(\left(p_{j}^{\prime} \wedge \varphi_{j}^{\prime}\right)\right. \\
& S_{5}, S_{1} \quad \mathrm{~g} \quad p_{1} \wedge \varphi_{1}: S_{1} \\
& p_{5} \wedge \varphi_{5}: S_{5}
\end{aligned}
$$




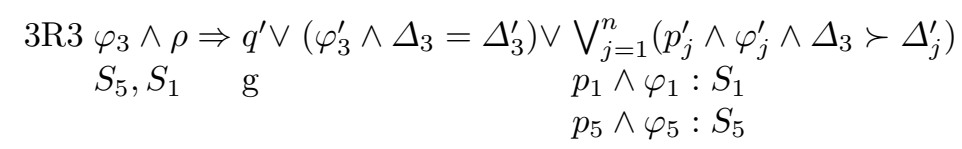

In $3 R_{3}$, we have $\Delta_{3} \succ_{S_{1}} \Delta_{1}$ and $\Delta_{3} \succ_{S_{5}} \Delta_{5}$. The validity of the former is trivial. The validity of latter depends on $D e c_{y}$. In $S_{5}$, we have $D e c_{y}=1$ meaning $y>y^{\prime}$. The values of $\Delta_{3}$ and $\Delta_{5}$ depend on the values of $y$. In this case, we have $\Delta_{3} \succ_{S_{5}} \Delta_{5}$ and the condition $\bigvee_{j=1}^{n}\left(p_{j}^{\prime} \wedge \varphi_{j}^{\prime} \wedge \Delta_{3} \succ \Delta_{j}^{\prime}\right)$ holds in $3 R_{3}$.

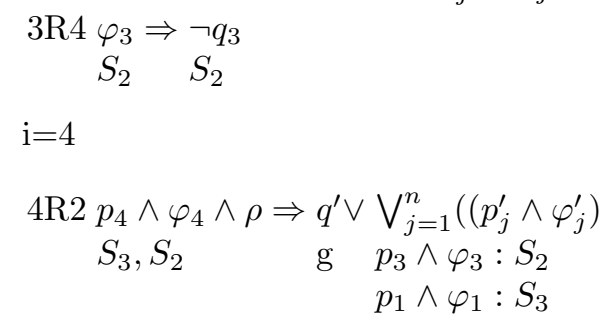
$4 \mathrm{R} 3 \varphi_{4} \wedge \rho \Rightarrow q^{\prime} \vee\left(\varphi_{4}^{\prime} \wedge \Delta_{4}=\Delta_{4}^{\prime}\right) \vee \bigvee_{j=1}^{n}\left(p_{j}^{\prime} \wedge \varphi_{j}^{\prime} \wedge \Delta_{4} \succ \Delta_{j}^{\prime}\right)$ $S_{3}, S_{2} \quad \mathrm{~g} \quad p_{3} \wedge \varphi_{3}: S_{2}$ $p_{2} \wedge \varphi_{2}: S_{3}$

$4 \mathrm{R} 4 \varphi_{4} \Rightarrow \neg q_{4}$

$S_{4} \quad S_{4}$

$\mathrm{i}=5$

$\begin{array}{cl}5 \mathrm{R} 2 p_{5} \wedge \varphi_{5} \wedge \rho \Rightarrow & q^{\prime} \vee \\ S_{4} & \bigvee_{j=1}^{n}\left(\left(p_{j}^{\prime} \wedge \varphi_{j}^{\prime}\right)\right. \\ & \mathrm{g} \quad p_{4} \wedge \varphi_{4}: S_{4}\end{array}$

$5 \mathrm{R} 3 \varphi_{5} \wedge \rho \Rightarrow q^{\prime} \vee\left(\varphi_{5}^{\prime} \wedge \Delta_{5}=\Delta_{5}^{\prime}\right) \vee \bigvee_{j=1}^{n}\left(p_{j}^{\prime} \wedge \varphi_{j}^{\prime} \wedge \Delta_{5} \succ \Delta_{j}^{\prime}\right)$

$S_{4} \quad \mathrm{~g} \quad p_{4} \wedge \varphi_{4}: S_{4}$

$5 \mathrm{R} 4 \varphi_{5} \Rightarrow \neg q_{5}$

$S_{5} \quad S_{5}$

$\mathrm{i}=6$

$6 \mathrm{R} 2 p_{7} \wedge \varphi_{6} \wedge \rho \Rightarrow q^{\prime} \vee \bigvee_{j=1}^{n}\left(\left(p_{j}^{\prime} \wedge \varphi_{j}^{\prime}\right)\right.$
$S_{4}$
$p_{4} \wedge \varphi_{4}: S_{4}$

$6 \mathrm{R} 3 \varphi_{6} \wedge \rho \Rightarrow q^{\prime} \vee\left(\varphi_{6}^{\prime} \wedge \Delta_{6}=\Delta_{6}^{\prime}\right) \vee \bigvee_{j=1}^{n}\left(p_{j}^{\prime} \wedge \varphi_{j}^{\prime} \wedge \Delta_{6} \succ \Delta_{j}^{\prime}\right)$

$S_{4} \quad p_{4} \wedge \varphi_{4}: S_{4}$

$6 \mathrm{R} 4 \varphi_{6} \quad \Rightarrow \neg q_{6}$

$S_{7}, S_{6} \quad S_{7}, S_{6}$

$\mathrm{i}=7$

$\begin{array}{cc}7 \mathrm{R} 2 p_{7} \wedge \varphi_{7} \wedge \rho \Rightarrow q^{\prime} \vee & \bigvee_{j=1}^{n}\left(\left(p_{j}^{\prime} \wedge \varphi_{j}^{\prime}\right)\right. \\ S_{6} & p_{6} \wedge \varphi_{6}: S_{6}\end{array}$

$7 \mathrm{R} 3 \varphi_{7} \wedge \rho \Rightarrow q^{\prime} \vee\left(\varphi_{7}^{\prime} \wedge \Delta_{7}=\Delta_{7}^{\prime}\right) \vee \bigvee_{j=1}^{n}\left(p_{j}^{\prime} \wedge \varphi_{j}^{\prime} \wedge \Delta_{7} \succ \Delta_{j}^{\prime}\right)$

$S_{6} \quad p_{6} \wedge \varphi_{6}: S_{6}$ 
$7 \mathrm{R} 4 \varphi_{7} \Rightarrow \neg q_{7}$

$S_{3} \quad S_{3}$

This means that every state in the transitions satisfies the premisses of the rule, therefore we can reach the goal state: $a t_{-} l_{3}$ with these transitions, and the liveness property holds.

\section{A.3 Example 3: UP-DOWN}

The goal, the compassion requirements and the auxiliary constructs for example 3 are listed as follows.

\begin{tabular}{|c|c|c|c|}
\hline $\mathrm{p}: a t_{-} l_{0} \wedge a t \_m_{0}$ & q: $a t_{-} l_{4}$ & & \\
\hline$\varphi_{1}: a t_{-} l_{2} \wedge a t_{-} m_{1} \wedge x>0 \wedge y=0$ & $p_{1}: 1$ & $q_{1}: \neg a t_{-} l_{2}$ & $\Delta_{1}:[1]$ \\
\hline$\varphi_{2}: a t_{-} l_{3} \wedge a t_{-} m_{1} \wedge x>0 \wedge y>0$ & $p_{2}: 1$ & $q_{2}: \neg a t_{-} l_{3}$ & $\Delta_{2}:[2, \mathrm{y}, 0]$ \\
\hline$\varphi_{3}: a t_{-} l_{2} \wedge a t \_m_{1} \wedge x>0 \wedge y>0$ & $p_{3}: 1$ & $q_{3}: \neg a t_{-} l_{2}$ & $\Delta_{3}:[2, \mathrm{y}, 1]$ \\
\hline$\varphi_{4}: a t_{-} l_{0} \wedge a t_{-} m_{1} \wedge x>0 \wedge y>0$ & $p_{4}: 1$ & $q_{4}: \neg a t_{-} l_{0}$ & $\Delta_{4}:[3]$ \\
\hline$\varphi_{5}: a t_{-} l_{1} \wedge a t_{-} m_{1} \wedge x>0 \wedge y>0$ & $p_{5}: 1$ & $q_{5}: \neg a t_{-} l_{1}$ & $\Delta_{5}:[4]$ \\
\hline$\varphi_{6}: a t_{-} l_{0,1} \wedge a t \_m_{0} \wedge x=0 \wedge y>0$ & $p_{6}: 1$ & $q_{6}: \neg a t_{-} m_{0}$ & $\Delta_{6}:[5]$ \\
\hline$\varphi_{7}: a t_{-} l_{0} \wedge a t_{-} m_{0} \wedge x=0 \wedge y>0$ & $p_{7}: 1$ & $q_{7}: \neg a t_{-} l_{0}$ & $\Delta_{7}:[6]$ \\
\hline$\varphi_{8}: a t_{l} l_{1} \wedge a t \_m_{0} \wedge x=0 \wedge y>0$ & $p_{8}: 1$ & $q_{8}: \neg a t_{-} l_{1}$ & $\Delta_{8}:[7]$ \\
\hline
\end{tabular}

We prove that the premisses of the rule C-RESPONSE hold when using the above auxiliary constructs as follows.

$$
\begin{aligned}
\mathrm{R} 1 \mathrm{p} \Rightarrow \mathrm{q} \vee & \bigvee_{j=1}^{n}\left(p_{j} \wedge \varphi_{j}\right) \\
S_{10}, S_{8} & p_{6} \wedge \varphi_{6}: S_{10}, S_{8} \\
& p_{7} \wedge \varphi_{7}: S_{10}, S_{8}
\end{aligned}
$$

This means that the initial states $S_{10}, S_{8}$ which satisfy $p: a t_{-} l_{0} \wedge a t_{-} m_{0}$ can be covered by the set of states specified on the right hand side.

$$
\begin{aligned}
& \mathrm{i}=1 \\
& 1 \mathrm{R} 2 p_{1} \wedge \varphi_{1} \wedge \rho \Rightarrow q^{\prime} \vee \bigvee_{j=1}^{n}\left(\left(p_{j}^{\prime} \wedge \varphi_{j}^{\prime}\right)\right. \\
& \mathrm{g} \quad \mathrm{g} \\
& \operatorname{1R} 3 \varphi_{1} \wedge \rho \Rightarrow q^{\prime} \vee\left(\varphi_{1} \wedge \Delta_{1}=\Delta_{1}^{\prime}\right) \vee \bigvee_{j=1}^{n}\left(p_{j}^{\prime} \wedge \varphi_{j}^{\prime} \wedge \Delta_{1} \succ \Delta_{j}^{\prime}\right) \\
& \mathrm{g} \quad \mathrm{g} \\
& \operatorname{1R} 4 \varphi_{1} \Rightarrow \neg q_{1} \\
& S_{1} \quad S_{1} \\
& \mathrm{i}=2 \\
& 2 \mathrm{R} 2 p_{2} \wedge \varphi_{2} \wedge \rho \Rightarrow q^{\prime} \vee \bigvee_{j=1}^{n}\left(\left(p_{j}^{\prime} \wedge \varphi_{j}^{\prime}\right)\right. \\
& S_{3}, S_{1} \quad p_{3} \wedge \varphi_{3}: S_{3} \\
& p_{1} \wedge \varphi_{1}: S_{1}
\end{aligned}
$$




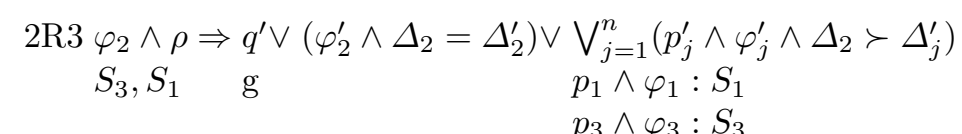

$2 \mathrm{R} 4 \varphi_{2} \quad \Rightarrow \neg q_{2}$

$S_{3}, S_{1} \quad S_{3}, S_{1}$

$\mathrm{i}=3$

$3 \mathrm{R} 2 p_{3} \wedge \varphi_{3} \wedge \rho \Rightarrow q^{\prime} \vee \bigvee_{j=1}^{n}\left(\left(p_{j}^{\prime} \wedge \varphi_{j}^{\prime}\right)\right.$

$S_{2} \quad$ g $p_{2} \wedge \varphi_{2}: S_{2}$

$3 \mathrm{R} 3 \varphi_{3} \wedge \rho \Rightarrow q^{\prime} \vee\left(\varphi_{3}^{\prime} \wedge \Delta_{3}=\Delta_{3}^{\prime}\right) \vee \bigvee_{j=1}^{n}\left(p_{j}^{\prime} \wedge \varphi_{j}^{\prime} \wedge \Delta_{3} \succ \Delta_{j}^{\prime}\right)$

$S_{2} \quad \mathrm{~g} \quad p_{2} \wedge \varphi_{2}: S_{2}$

$3 \mathrm{R} 4 \varphi_{3} \quad \Rightarrow \neg q_{3}$

$S_{3}, S_{4} \quad S_{3}, S_{4}$

$\mathrm{i}=4$

$4 \mathrm{R} 2 p_{4} \wedge \varphi_{4} \wedge \rho \Rightarrow q^{\prime} \bigvee \bigvee_{j=1}^{n}\left(\left(p_{j}^{\prime} \wedge \varphi_{j}^{\prime}\right)\right.$

$S_{4} \quad$ g $p_{3} \wedge \varphi_{3}: S_{4}$

$4 \mathrm{R} 3 \varphi_{4} \wedge \rho \Rightarrow q^{\prime} \vee\left(\varphi_{4}^{\prime} \wedge \Delta_{4}=\Delta_{4}^{\prime}\right) \vee \bigvee_{j=1}^{n}\left(p_{j}^{\prime} \wedge \varphi_{j}^{\prime} \wedge \Delta_{4} \succ \Delta_{j}^{\prime}\right)$

$S_{4} \quad \mathrm{~g} \quad p_{3} \wedge \varphi_{3}: S_{4}$

$4 \mathrm{R} 4 \varphi_{4} \quad \Rightarrow \neg q_{4}$

$S_{5} S_{6} \quad S_{5} S_{6}$

$\mathrm{i}=5$

$5 \mathrm{R} 2 p_{5} \wedge \varphi_{5} \wedge \rho \Rightarrow q^{\prime} \bigvee \bigvee_{j=1}^{n}\left(\left(p_{j}^{\prime} \wedge \varphi_{j}^{\prime}\right)\right.$

$S_{6} \quad$ g $p_{4} \wedge \varphi_{4}: S_{6}$

$5 \mathrm{R} 3 \varphi_{5} \wedge \rho \Rightarrow q^{\prime} \vee\left(\varphi_{5}^{\prime} \wedge \Delta_{5}=\Delta_{5}^{\prime}\right) \vee \bigvee_{j=1}^{n}\left(p_{j}^{\prime} \wedge \varphi_{j}^{\prime} \wedge \Delta_{5} \succ \Delta_{j}^{\prime}\right)$

$S_{6} \quad \mathrm{~g} \quad p_{4} \wedge \varphi_{4}: S_{6}$

$5 \mathrm{R} 4 \varphi_{5} \Rightarrow \neg q_{5}$

$S_{7} \quad S_{7}$

$\mathrm{i}=6$

$6 \mathrm{R} 2 p_{7} \wedge \varphi_{6} \wedge \rho \Rightarrow q^{\prime} \bigvee \bigvee_{j=1}^{n}\left(\left(p_{j}^{\prime} \wedge \varphi_{j}^{\prime}\right)\right.$

$S_{4}, S_{7}, S_{5} \quad p_{3} \wedge \varphi_{3}: S_{5}$

$p_{4} \wedge \varphi_{4}: S_{5}$

$p_{5} \wedge \varphi_{5}: S_{7}$

$6 \mathrm{R} 3 \varphi_{6} \wedge \rho \Rightarrow q^{\prime} \vee\left(\varphi_{6}^{\prime} \wedge \Delta_{6}=\Delta_{6}^{\prime}\right) \vee \bigvee_{j=1}^{n}\left(p_{j}^{\prime} \wedge \varphi_{j}^{\prime} \wedge \Delta_{6} \succ \Delta_{j}^{\prime}\right)$

$S_{4}, S_{7}, S_{5}$

$p_{3} \wedge \varphi_{3}: S_{5}$

$p_{4} \wedge \varphi_{4}: S_{5}$

$p_{5} \wedge \varphi_{5}: S_{7}$

$6 \mathrm{R} 4 \varphi_{6} \quad \Rightarrow \neg q_{6}$

$S_{8}, S_{9}, S_{10} \quad S_{8}, S_{9}, S_{10}$ 
$\mathrm{i}=7$

$$
\begin{gathered}
7 \mathrm{R} 2 p_{7} \wedge \varphi_{7} \wedge \rho \Rightarrow q^{\prime} \vee \bigvee_{j=1}^{n}\left(\left(p_{j}^{\prime} \wedge \varphi_{j}^{\prime}\right)\right. \\
S_{9} \quad p_{6} \wedge \varphi_{6}: S_{9}
\end{gathered}
$$$$
7 \mathrm{R} 3 \varphi_{7} \wedge \rho \Rightarrow q^{\prime} \vee\left(\varphi_{7}^{\prime} \wedge \Delta_{7}=\Delta_{7}^{\prime}\right) \vee \bigvee_{j=1}^{n}\left(p_{j}^{\prime} \wedge \varphi_{j}^{\prime} \wedge \Delta_{7} \succ \Delta_{j}^{\prime}\right)
$$$$
S_{9} \quad p_{6} \wedge \varphi_{6}: S_{9}
$$

$7 \mathrm{R} 4 \varphi_{7} \quad \Rightarrow \neg q_{7}$

$S_{8}, S_{10} \quad S_{8}, S_{10}$

$\mathrm{i}=8$

$8 \mathrm{R} 2 p_{7} \wedge \varphi_{7} \wedge \rho \Rightarrow q^{\prime} \vee \bigvee_{j=1}^{n}\left(\left(p_{j}^{\prime} \wedge \varphi_{j}^{\prime}\right)\right.$

$S_{8} \quad p_{7} \wedge \varphi_{7}: S_{8}$

$8 \mathrm{R} 3 \varphi_{7} \wedge \rho \Rightarrow q^{\prime} \vee\left(\varphi_{7}^{\prime} \wedge \Delta_{7}=\Delta_{7}^{\prime}\right) \vee \bigvee_{j=1}^{n}\left(p_{j}^{\prime} \wedge \varphi_{j}^{\prime} \wedge \Delta_{7} \succ \Delta_{j}^{\prime}\right)$

$S_{8}$

$p_{7} \wedge \varphi_{7}: S_{8}$

8R4 $\varphi_{7} \Rightarrow \neg q_{7}$

$S_{9} \quad S_{9}$

This means that every state in the transitions satisfies the premisses of the rule, therefore we can reach the goal state: $a t_{-} l_{4}$ with these transitions, and the liveness property holds. 